\title{
Synthesis of 2-oxazolidinones from $\beta$-lactams: Stereospecific total synthesis of (-)-Cytoxazone and all of its stereoisomers.
}

Rajesh Kumar Mishra, Cristina M. Coates, Kevin D. Revell and Edward Turos*

Center for Molecular Diversity in Drug Design, Discovery, and Delivery, Department of Chemistry,

University of South Florida, 4202 East Fowler Avenue, Tampa, Florida 33620 USA.

eturos@shell.cas.usf.edu

\section{Supporting Information}


General: THF was distilled from sodium/benzophenone. $\mathrm{CH}_{2} \mathrm{Cl}_{2}, \mathrm{Et}_{3} \mathrm{~N}$ and diisopropylamine were freshly distilled from $\mathrm{CaH}_{2}$. All other solvents and reagents were obtained from commercial sources and used without further purification. Organic extracts were dried over anhydrous $\mathrm{Na}_{2} \mathrm{SO}_{4}$, filtered, and evaporated by rotary evaporation. Non-volatile oils and solids were vacuum dried prior to characterization. Optical rotations were measured on a digital polarimeter. Melting points are uncorrected. Flash column chromatography was performed using commercial grades of silica gel finer than $220 \mathrm{mesh}$. Analytical thin layer chromatography was performed on precoated Merck silica gel $60 \mathrm{~F}_{254}$ plates, and compounds were visualized by UV illumination ( $254 \mathrm{~nm}$ ).

( \pm )-(2S, 3R)-Methyl 2-hydroxy-3-(4-methoxyphenylamino)-3-phenylpropanoate (2):

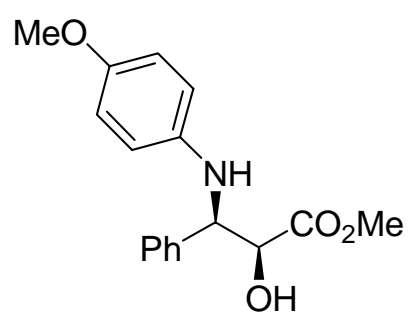

2

Chlorotrimethylsilane $(11.6 \mathrm{~mL}, 93 \mathrm{mmol})$ was added dropwise at $0{ }^{\circ} \mathrm{C}$ to a stirred solution of hydroxy $\beta$-lactam $\mathbf{1}(5 \mathrm{~g}, 18.6 \mathrm{mmol})$ in anhydrous methanol $(100 \mathrm{~mL})$. The reaction mixture was refluxed for 5 hours, cooled to room temperature and the solvent was removed under vacuum. The crude residue was dissolved in water and neutralized with $5 \%$ aqueous $\mathrm{NaHCO}_{3}$, followed by extraction with ethyl acetate $(3 \times 100 \mathrm{~mL})$. The combined organic extracts were dried over $\mathrm{Na}_{2} \mathrm{SO}_{4}$, concentrated under vacuum and purified by flash column chromatography to afford the title compound $2(5.3 \mathrm{~g}, 95 \%)$ as a white solid; mp $69-71{ }^{\circ} \mathrm{C} ;{ }^{1} \mathrm{H} \mathrm{NMR}\left(250 \mathrm{MHz}, \mathrm{CDCl}_{3}\right)$ $\delta$ : 7.38-7.28 (5H, m), $6.71(2 \mathrm{H}, \mathrm{d}, J=8.8 \mathrm{~Hz}), 6.55(2 \mathrm{H}, \mathrm{d}, J=8.8 \mathrm{~Hz}), 4.88(1 \mathrm{H}, \mathrm{d}, J=2.5 \mathrm{~Hz}), 4.52(1 \mathrm{H}, \mathrm{d}, J=2.8 \mathrm{~Hz}), 3.81(3 \mathrm{H}, \mathrm{s})$, $3.71(3 \mathrm{H}, \mathrm{s}) ;{ }^{13} \mathrm{C} \mathrm{NMR}\left(62.5 \mathrm{MHz}, \mathrm{CDCl}_{3}\right) \delta$ : 173.4, 152.3, 140.4, 139.4, 128.5, 127.5, 127.0, 115.4,114.7, 74.7, 60.0, 55.6, 53.0. 


\section{$( \pm)-(4 R, 5 S)-M e t h y l$ 3-(4-methoxyphenyl)-2-oxo-4-phenyloxazolidine-5-carboxylate (3):}

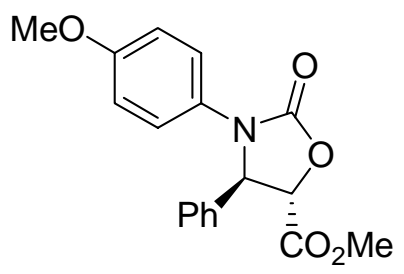

3

To a stirred solution of amino ester $2(4 \mathrm{~g}, 13.2 \mathrm{mmol})$ in anhydrous $\mathrm{CH}_{2} \mathrm{Cl}_{2}(100 \mathrm{~mL})$ at $0{ }^{\circ} \mathrm{C}$ was added Hunig's base $(5.6 \mathrm{~mL}, 39.8$ $\mathrm{mmol})$ dropwise and the reaction mixture was stirred for 15 minutes at $0{ }^{\circ} \mathrm{C}$. A solution of triphosgene $(4.7 \mathrm{~g}, 15.8 \mathrm{mmol})$ in anhydrous $\mathrm{CH}_{2} \mathrm{Cl}_{2}(50 \mathrm{~mL})$ was added dropwise and the reaction mixture was allowed to warm to room temperature and stirred for 3 hours. The mixture was diluted with $\mathrm{CH}_{2} \mathrm{Cl}_{2}$, washed with water, brine, dried over $\mathrm{Na}_{2} \mathrm{SO}_{4}$ and the solvent was removed by rotary evaporation. The residue was purified by flash column chromatography to yield oxazolidinone $3(3.4 \mathrm{~g}, 80 \%)$ as a white solid; $\mathrm{mp}$

109-111 ${ }^{\circ} \mathrm{C} .{ }^{1} \mathrm{H}$ NMR $\left(250 \mathrm{MHz}, \mathrm{CDCl}_{3}\right) \delta: 7.40-7.27(7 \mathrm{H}, \mathrm{m}), 6.81(2 \mathrm{H}, \mathrm{dd}, J=7.0,2.3 \mathrm{~Hz}), 5.38(1 \mathrm{H}, \mathrm{d}, J=4.5 \mathrm{~Hz}), 4.80(1 \mathrm{H}, \mathrm{d}, J=$ $4.3 \mathrm{~Hz}), 3.92(3 \mathrm{H}, \mathrm{s}), 3.75(3 \mathrm{H}, \mathrm{s}) ;{ }^{13} \mathrm{C} \mathrm{NMR}\left(62.5 \mathrm{~Hz} \mathrm{MHz}, \mathrm{CDCl}_{3}\right)$ 8: 168.8, 157.1, 154.4, 137.6, 129.4, 129.3, 129.2, 126.3, 123.1, 114.3, 76.5, 64.1, 55.4, 53.2. 


\section{$( \pm)-(4 R, 5 S)-M e t h y l$ 2-oxo-4-phenyloxazolidine-5-carboxylate:}

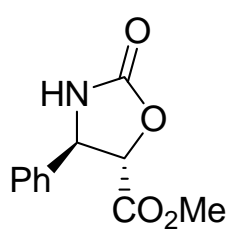

4

To a stirred solution of oxazolidinone $3(2.0 \mathrm{~g}, 6.1 \mathrm{mmol})$ in $\mathrm{CH}_{3} \mathrm{CN}$ and water $(50 \mathrm{~mL}, 7: 3)$ at $0^{\circ} \mathrm{C}$ was added drop wise a solution of ceric ammonium nitrate $(10.0 \mathrm{~g}, 18.2 \mathrm{mmol})$ in $\mathrm{CH}_{3} \mathrm{CN}$ and water $(50 \mathrm{~mL}, 1: 10 \mathrm{v}: \mathrm{v})$ over a period of 30 minutes. The mixture was allowed to warm to room temperature and stirred for another two hours. The reaction mixture was extracted with ethyl acetate $(3 \times 50$ $\mathrm{mL}$ ) and the combined organic extracts were washed with $10 \%$ aqueous $\mathrm{NaHCO}_{3}$ solution $(3 \times 100 \mathrm{~mL})$, brine, dried over $\mathrm{Na}_{2} \mathrm{SO}_{4}$ and concentrated by rotary evaporation. The crude product was purified by flash column chromatography to afford the compound 4 (946 mg, 70\%) as a white solid; mp 112-115 ${ }^{\circ} \mathrm{C} .{ }^{1} \mathrm{H}$ NMR $\left(250 \mathrm{MHz}, \mathrm{CDCl}_{3}\right) \delta$ : 7.45-7.41 $(5 \mathrm{H}, \mathrm{m}), 6.00(1 \mathrm{H}, \mathrm{br} \mathrm{s}), 5.02(1 \mathrm{H}, \mathrm{d}, J=$ $5.3 \mathrm{~Hz}), 4.80(1 \mathrm{H}, \mathrm{d}, J=5.3 \mathrm{~Hz}), 3.90(3 \mathrm{H}, \mathrm{s}) ;{ }^{13} \mathrm{C} \mathrm{NMR}\left(62.5 \mathrm{~Hz} \mathrm{MHz}, \mathrm{CDCl}_{3}\right)$ 8: 168.8, 158.3, 138.9, 129.2, 129.0, 125.8, 80.2, 59.1, 53.13. 


\section{( \pm )-(4R, 5S)-5-(Hydroxymethyl)-4-phenyloxazolidin-2-one (5):}

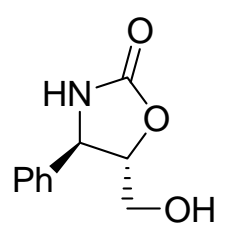

5

To a stirred solution of methyl ester $4(0.8 \mathrm{~g}, 3.6 \mathrm{mmol})$ in aqueous $\mathrm{THF}(25 \mathrm{~mL})$ at $0{ }^{\circ} \mathrm{C}$ was added $\mathrm{NaBH}_{4}(0.4 \mathrm{~g}, 10.8 \mathrm{mmol})$ in one portion and the reaction mixture was allowed to warm to room temperature. The reaction mixture was stirred for six hours and quenched with saturated aqueous $\mathrm{NH}_{4} \mathrm{Cl}$ solution and extracted with ethyl acetate $(3 \mathrm{x} 50 \mathrm{~mL})$. The combined organic extracts were washed with brine, dried over $\mathrm{Na}_{2} \mathrm{SO}_{4}$ and concentrated by rotary evaporation. The crude product was purified by flash column chromatography to afford alcohol $5(0.64 \mathrm{~g}, 92 \%)$ as a white solid; mp 106-108 ${ }^{\circ} \mathrm{C} .{ }^{1} \mathrm{H} \mathrm{NMR}\left(250 \mathrm{MHz}\right.$, acetone-d $\left.{ }_{6}\right) \delta: 7.44-7.35(5 \mathrm{H}$, m), $7.09\left(1 \mathrm{H}\right.$, br s), $4.87(1 \mathrm{H}, \mathrm{d}, J=6.3 \mathrm{~Hz}), 4.45(1 \mathrm{H}, \mathrm{br} \mathrm{s}), 4.29(1 \mathrm{H}, \mathrm{dt}, J=2.5,6.3 \mathrm{~Hz}), 3.80(2 \mathrm{H}, \mathrm{ddd}, J=4.0,6.3,12.5 \mathrm{~Hz}) ;{ }^{13} \mathrm{C}$ NMR $\left(62.5 \mathrm{MHz}\right.$, acetone- $\left.\mathrm{d}_{6}\right) \delta: 159.0,142.2,129.7,128.9,127.1,85.4,62.5,58.1$. 
( \pm )-((4R, 5S)-2-Oxo-4-phenyloxazolidin-5-yl)methyl methanesulfonate (6):

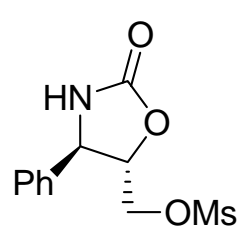

6

To a solution of alcohol $5(0.50 \mathrm{~g}, 2.5 \mathrm{mmol})$ in freshly distilled $\mathrm{CH}_{2} \mathrm{Cl}_{2}(50 \mathrm{~mL})$ at $0{ }^{\circ} \mathrm{C}$ was added triethylamine $(1.0 \mathrm{~mL}, 7.7 \mathrm{mmol})$ under the atmosphere of nitrogen. The reaction was stirred for 30 minutes, followed by addition of methanesulfonyl chloride (300 $\mu \mathrm{L}$ $3.8 \mathrm{mmol}$ ). The reaction mixture was stirred for six hours at room temperature. It was diluted with $\mathrm{CH}_{2} \mathrm{Cl}_{2}$, washed with water, brine, dried over $\mathrm{Na}_{2} \mathrm{SO}_{4}$ and concentrated by rotary evaporation. The crude product was purified by flash column chromatography to afford mesylate 6 (0.68 g, 98\%) as a yellow oil. ${ }^{1} \mathrm{H}$ NMR (250 MHz, $\left.\mathrm{CDCl}_{3}\right)$ 8: 7.34-7.24 (5H, m), $7.04(1 \mathrm{H}, \mathrm{br} \mathrm{s}), 4.71(1 \mathrm{H}, \mathrm{d}, \mathrm{J}=6.5 \mathrm{~Hz})$, 4.47-4.35 (3H, m), $3.06(3 \mathrm{H}, \mathrm{s})$. 
( \pm )-(4R, 5S)-5-(Azidomethyl)-4-phenyloxazolidin-2-one (7):

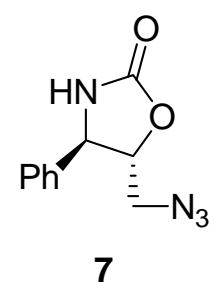

A mixture of mesylate $6(0.5 \mathrm{~g}, 1.8 \mathrm{mmol})$ and $\mathrm{NaN}_{3}(0.14 \mathrm{~g}, 2.2 \mathrm{mmol})$ in anhydrous DMF $(10 \mathrm{~mL})$ was refluxed for $12 \mathrm{hours}$ under the atmosphere of nitrogen. The reaction mixture was cooled to room temperature and dilute with ice cold water. The solution was extracted with ethyl acetate $(3 \times 50 \mathrm{~mL})$ and the combine organic extracts were dried over $\mathrm{Na}_{2} \mathrm{SO}_{4}$. The solvent was removed under vacuum and the crude product was purified by flash column chromatography to afford azide 7 (0. $29 \mathrm{~g}$, $72 \%)$ as a white solid; mp 82 $85{ }^{\circ} \mathrm{C} ;{ }^{1} \mathrm{H} \mathrm{NMR}\left(250 \mathrm{MHz}, \mathrm{CDCl}_{3}\right) \delta: 7.49-7.29(5 \mathrm{H}, \mathrm{m}), 6.29(1 \mathrm{H}$, br s), $4.65(1 \mathrm{H}, \mathrm{d}, J=6.0 \mathrm{~Hz}), 4.52-4.46(1 \mathrm{H}, \mathrm{m}), 3.75-3.52(2 \mathrm{H}$, $\mathrm{m}) ;{ }^{13} \mathrm{C} \mathrm{NMR}\left(62.5 \mathrm{~Hz} \mathrm{MHz}, \mathrm{CDCl}_{3}\right) \delta: 158.4,138.6,129.3,129.0,126.1,82.7,58.8,51.9$. 
( \pm )- ((4R, 5R)-2-Oxo-4-phenyloxazolidin-5-yl)methyl)acetamide (8):

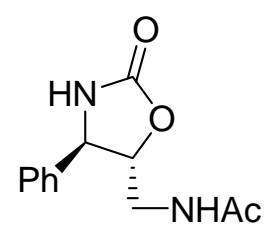

8

To a solution of azide $7(0.1 \mathrm{~g}, 0.4 \mathrm{mmol})$ in ethyl acetate $(10 \mathrm{~mL})$ was added a catalytic amount of $10 \% \mathrm{Pd} / \mathrm{C}$. The reaction mixture was stirred at room temperature for 12 hours under the atmosphere of hydrogen. The reaction mixture was evacuated and flushed with nitrogen. The mixture was cooled to $0^{\circ} \mathrm{C}$ and pyridine $(147 \mu \mathrm{L}, 1.8 \mathrm{mmol})$ and acetic anhydride $(173 \mu \mathrm{L}, 1.8 \mathrm{mmol})$ were added sequentially and further stirred for 6 hours. The solution was diluted with ethyl acetate and washed with water, brine, dried over $\mathrm{Na}_{2} \mathrm{SO}_{4}$ and concentrated by rotary evaporation. The crude product was purified by flash column chromatography to afford $\mathbf{8}$ ( $0.08 \mathrm{~g}$, 75\%) as a white solid; mp 118-120 ${ }^{\circ} \mathrm{C} .{ }^{1} \mathrm{H}$ NMR (250 MHz, $\left.\mathrm{CDCl}_{3}\right): \delta 7.40-7.31(5 \mathrm{H}, \mathrm{m}), 6.23(1 \mathrm{H}, \mathrm{br} \mathrm{s}), 5.60(1 \mathrm{H}, \mathrm{br} \mathrm{s}), 4.64(1 \mathrm{H}, \mathrm{d}$, $J=7.3 \mathrm{~Hz}), 4.44-4.38(1 \mathrm{H}, \mathrm{m}), 3.67-3.57(2 \mathrm{H}, \mathrm{m}), 2.01(3 \mathrm{H}, \mathrm{s}) ;{ }^{13} \mathrm{C} \mathrm{NMR}\left(62.5 \mathrm{~Hz}, \mathrm{CDCl}_{3}\right): \delta 171.1,158.6,138.4,129.2,128.9,128.7$, 128.6, 126.0, 83.7, 58.6, 40.2, 23.1. 


\section{Synthesis of Optically Pure Hydroxy $\beta$-Lactam 13:}

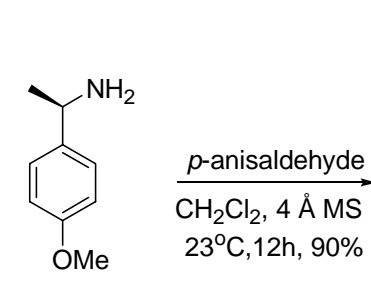

20

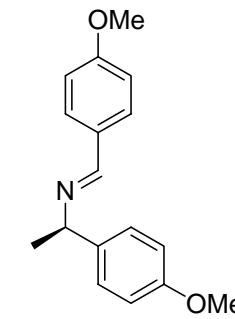

21

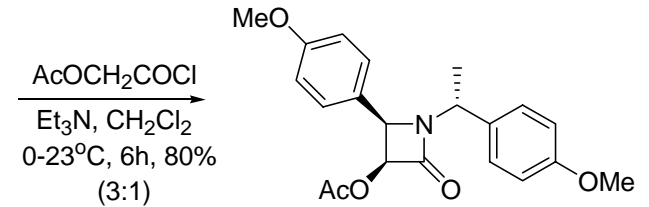

22a

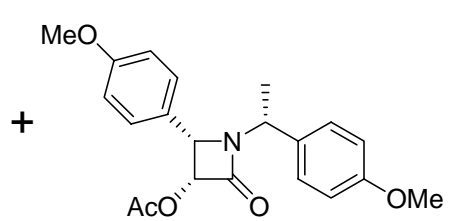

$22 b$

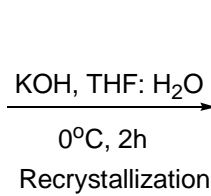

$60 \%$

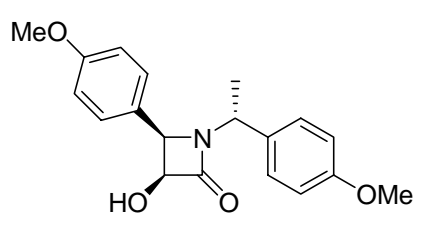

13

The above reaction scheme was used to prepare enantiomerically-pure lactam 13 in a manner similar to that previously reported (see Brown, S.; Jordan, A. M.; Lawrence, N. J.; Pritchard, R. G.; McGown, A. T. Tetrahedron Lett. 1998, 39, 3559; Bourzat, J.D.; Commercon, A.; Tetrahedron Lett. 1993, 34, 6042; Ojima, I.; Habus, I.; Zhao, M.; Zucco, M.; Park, Y. H.; Sun, C. M.; Brigaud, T. Tetrahedron. 1992, 48, 6985). p-Anisaldehyde was combined with an equimolar amount of amine $\mathbf{2 0}$ to afford chiral imine 21, which was immediately subjected to Staudinger reaction with acetoxyacetyl chloride (one molar equivalent) and Et $\mathrm{t}_{3} \mathrm{~N}(3 \mathrm{molar}$ equivalents) in methylene chloride to afford azetidinones 22 in 80\% yield as a 3:1 diastereomeric mixture. Although the minor diastereomer could in principle be removed at this stage, it was easier to do the separation after the next step. Thus, the acetate functionality was hydrolyzed with one equivalent of $\mathrm{KOH}$ in THF: $\mathrm{H}_{2} \mathrm{O}(7: 3)$ and the resultant diastereomeric mixture of hydroxy $\beta$-lactams was recrystallized with EtOAc: hexane (7:3) to afford a 60\% yield of hydroxy $\beta$-lactam 13 as a single diastereomer. 
(E)-(R)-N-(4-Methoxybenzylidene)-1-(4-methoxyphenyl)ethanamine (21):

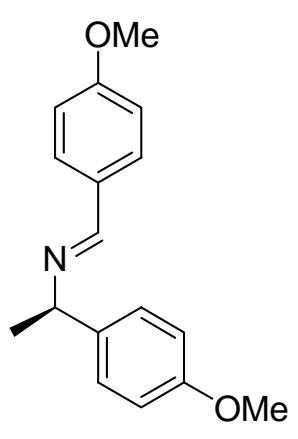

21

To a solution of 4-methoxybenzaldehyde $(10.0 \mathrm{~g}, 73.4 \mathrm{mmol})$ in $\mathrm{CH}_{2} \mathrm{Cl}_{2}(250 \mathrm{~mL})$ was added amine 20 (11.0 g, $\left.73.4 \mathrm{mmol}\right)$ and powdered $4 \AA$ molecular sieves $(5 \mathrm{~g})$. The reaction mixture was stirred for 12 hours at room temperature and then filtered through a sintered glass vacuum filtration funnel containing a $1 / 2$ " pad of Celite. The pad was washed three times with $\mathrm{CH}_{2} \mathrm{Cl}_{2}$ and the filtrate was concentrated to dryness by rotary evaporation to give imine 21 (17.7 g, 90\%) as an opalescent oil. This imine was used in the next step without further purification. ${ }^{1} \mathrm{H} \mathrm{NMR}\left(\mathrm{CDCl}_{3}, 250 \mathrm{MHz}\right): \delta 8.33(1 \mathrm{H}, \mathrm{s}), 7.78-7.74(2 \mathrm{H}, \mathrm{m}), 7.41-7.36(2 \mathrm{H}, \mathrm{m}), 6.97-6.90(4 \mathrm{H}, \mathrm{m})$, $4.52(1 \mathrm{H}, \mathrm{q}, J=6.5 \mathrm{~Hz}), 3.86(3 \mathrm{H}, \mathrm{s}), 3.83(3 \mathrm{H}, \mathrm{s}), 1.61(3 \mathrm{H}, \mathrm{d}, J=6.5 \mathrm{~Hz}) ;{ }^{13} \mathrm{C} \mathrm{NMR}\left(62.5 \mathrm{~Hz}, \mathrm{CDCl}_{3}\right): \delta 161.5,158.5,158.4,137.6$, $129.7,129.4,127.6,113.9,113.8,58.9,55.3,55.2,24.8$. 
3-Acetoxy-4-(4-methoxypheny)-1-[(R)-1-(4-methoxyphenyl)ethyl] azetidin-2-ones (22a and 22b):
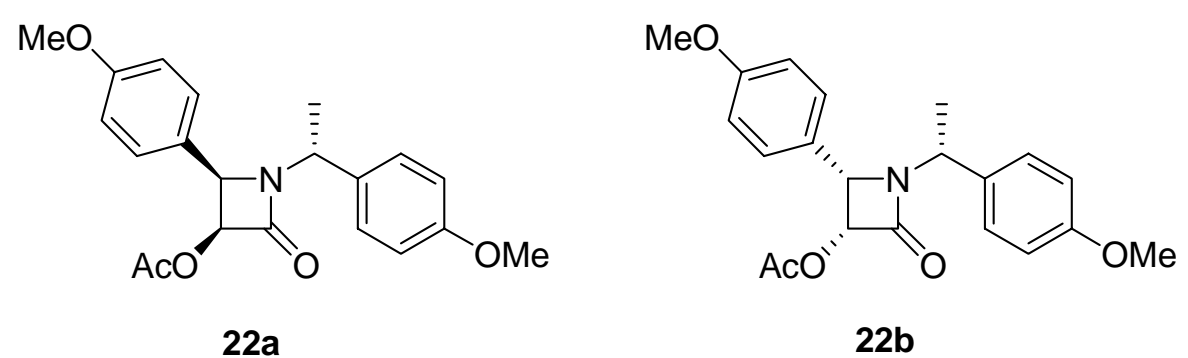

To a solution of imine $21(10 \mathrm{~g}, 37.1 \mathrm{mmol})$ in $\mathrm{CH}_{2} \mathrm{Cl}_{2}(250 \mathrm{~mL})$ was added triethylamine $(15.6 \mathrm{~mL}, 111.3 \mathrm{mmol})$ under a nitrogen atmosphere at room temperature. The reaction mixture was cooled to $0^{\circ} \mathrm{C}$ and a solution of acetoxyacetyl chloride $(5.1 \mathrm{~mL}, 44.5$ $\mathrm{mmol})$ in $\mathrm{CH}_{2} \mathrm{Cl}_{2}(100 \mathrm{~mL})$ was added dropwise over a period of one hour. The reaction mixture was stirred at room temperature for 12 hours and then diluted with $\mathrm{CH}_{2} \mathrm{Cl}_{2}$, washed with water, brine, dried over $\mathrm{Na}_{2} \mathrm{SO}_{4}$ and then concentrated by rotary evaporation to afford a brownish yellow oil. This oil was purified by flash column chromatography to afford a 3:1 diastereomeric mixture of $\beta$ lactams 22a and 22b (10.9 g, 80 \%). This mixture was carried forward to the next step without any further purification. 


\section{(3S, 4R)-3-Hydroxy-4(methoxyphenyl)-1-[(R)-1-(4-methoxyphenyl) ethyl] azetidin-2-one (13):}

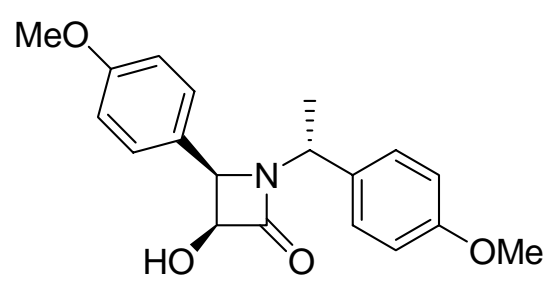

13

To a solution of solid $\mathrm{KOH}(2.7 \mathrm{~g}, 48.7 \mathrm{mmol})$ in THF: $\mathrm{H}_{2} \mathrm{O}(50 \mathrm{~mL}, 7: 3 \mathrm{v}: \mathrm{v})$ at $0^{\circ} \mathrm{C}$ was added dropwise a solution of a mixture $(3: 1$ molar ratio) of the two diastereomers $22 \mathrm{a}$ and $22 \mathbf{b}(6 \mathrm{~g}, 16.2 \mathrm{mmol})$ in THF $(25 \mathrm{~mL})$. The reaction mixture was stirred at $0^{\circ} \mathrm{C}$ for two hours and then diluted with water and extracted with ethyl acetate. The combined organic extracts were dried over $\mathrm{Na}_{2} \mathrm{SO}_{4}$ and then concentrated to dryness by rotary evaporation to give a white solid, which was recrystallized from $40 \mathrm{~mL}$ of a $3: 1 \mathrm{mixture}$ of ethyl acetate: hexane to give compound $\mathbf{1 3}(3.0 \mathrm{~g}, 58 \%)$ as a single diastereomer; mp $128-130{ }^{\circ} \mathrm{C} ;[\alpha]_{\mathrm{D}}{ }^{23}-227.0(\mathrm{c}=0.5 \mathrm{M}, \mathrm{CHCl}) ;{ }^{1} \mathrm{H}-\mathrm{NMR}$ $\left(250 \mathrm{MHz}, \mathrm{CDCl}_{3}\right) \delta: 7.32-7.17(4 \mathrm{H}, \mathrm{m}), 6.98-6.88(4 \mathrm{H}, \mathrm{m}), 5.06(1 \mathrm{H}, \mathrm{q}, J=7.5 \mathrm{~Hz}), 4.90(1 \mathrm{H}, \mathrm{dd}, J=5.0,7.5 \mathrm{~Hz}), 4.53(1 \mathrm{H}, \mathrm{d}, J=$ $5.0 \mathrm{~Hz}), 3.88(3 \mathrm{H}, \mathrm{s}), 3.83(3 \mathrm{H}, \mathrm{s}), 2.50(1 \mathrm{H}, \mathrm{br} \mathrm{s}), 1.40(3 \mathrm{H}, \mathrm{d}, J=7.3 \mathrm{~Hz}) ;{ }^{13} \mathrm{C}-\mathrm{NMR}\left(62.5 \mathrm{MHz}, \mathrm{CDCl}_{3}\right) \delta: 169.1,159.9,159.1$, $131.6,129.7,128.5,126.8,113.9,76.5,61.3,55.3,51.3,19.4$. 
(2S, 3R)-methyl 2-hydroxy-3-(4-methoxyphenyl)-3-(R)-(4-methoxyphenyl) ethyl amino) propionate (14):

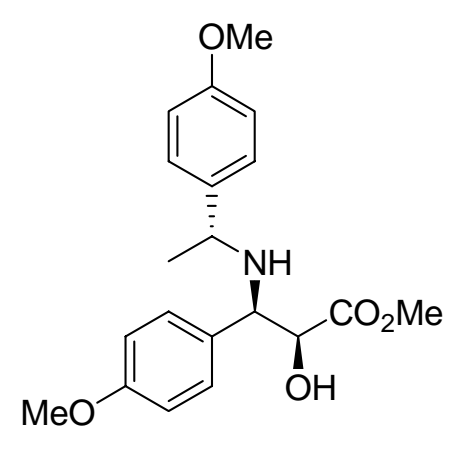

14

Compound 14 was synthesized from compound 13 employing the same procedure as described for compound 2. mp $88-90{ }^{\circ} \mathrm{C} ;[\alpha]_{\mathrm{D}}{ }^{23}$ $28.1\left(\mathrm{c}=0.5 \mathrm{M}, \mathrm{CHCl}_{3}\right) ;{ }^{1} \mathrm{H} \mathrm{NMR}\left(250 \mathrm{MHz}, \mathrm{CDCl}_{3}\right) \delta: 7.24(2 \mathrm{H}, \mathrm{d}, J=8.5 \mathrm{~Hz}), 7.17(2 \mathrm{H}, \mathrm{d}, J=8.5 \mathrm{~Hz}), 6.90(2 \mathrm{H}, \mathrm{d}, J=8.5 \mathrm{~Hz}), 6.85$ $(2 \mathrm{H}, \mathrm{d}, J=8.5 \mathrm{~Hz}), 4.26(1 \mathrm{H}, \mathrm{d}, J=3.3 \mathrm{~Hz}), 4.14(1 \mathrm{H}, \mathrm{d}, J=3.5 \mathrm{~Hz}), 3.85(3 \mathrm{H}, \mathrm{s}), 3.81(3 \mathrm{H}, \mathrm{s}), 3.79(3 \mathrm{H}, \mathrm{s}), 3.77(3 \mathrm{H}, \mathrm{s}), 3.63(1 \mathrm{H}, \mathrm{q}$, $J=5.5 \mathrm{~Hz}), 1.29(3 \mathrm{H}, \mathrm{d}, J=6.8 \mathrm{~Hz}) ;{ }^{13} \mathrm{C} \mathrm{NMR}\left(62.5 \mathrm{MHz}, \mathrm{CDCl}_{3}\right) \delta: 174.0,159.0,158.6,137.9,132.3,128.5,127.5,113.9,113.8$, $76.6,74.5,61.0,55.2,54.0,53.8,52.3,22.4$. 


\section{(2S, 3R)-methyl 3-(tert-butoxycarbonylamino-2-hydroxy-3-(4-methoxyphenyl)propanoate (15):}

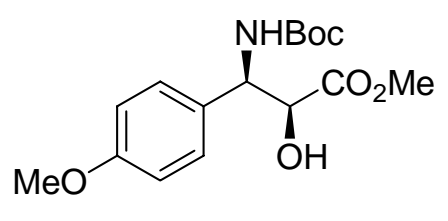

15

To a solution of compound $14(1.0 \mathrm{~g}, 2.7 \mathrm{mmol})$ in anhydrous methanol $(25 \mathrm{~mL})$ was added a catalytic amount of Pearlman's catalyst $\left[\mathrm{Pd}(\mathrm{OH})_{2} / \mathrm{C}\right]$ and the reaction mixture was stirred at room temperature for 12 hours under an atmosphere of hydrogen (balloon). The flask was evacuated and flushed with nitrogen. It was then charged with powdered $\mathrm{NaHCO}_{3}(0.7 \mathrm{~g}, 8.3 \mathrm{mmol})$ and $\mathrm{Boc} 2 \mathrm{O}(0.72 \mathrm{~g}, 3.3$ mmol). The resultant reaction mixture was sonicated at room temperature for six hours, then filtered through a sintered funnel containing a pad of Celite. The pad was washed three times with methanol and the filtrate was concentrated to dryness by rotary evaporation. The crude product was partitioned between water and $\mathrm{CH}_{2} \mathrm{Cl}_{2}$. and extracted with $\mathrm{CH}_{2} \mathrm{Cl}_{2}$. The combined organic extracts were washed with brine, dried over $\mathrm{Na}_{2} \mathrm{SO}_{4}$, and concentrated by rotary evaporation. The crude product was purified by flash chromatography to afford the compound $15(0.72 \mathrm{~g}, 80 \%)$ as a white solid; mp $110-112^{\circ} \mathrm{C} ;[\alpha]_{\mathrm{D}}{ }^{23}-3.8(\mathrm{c}=0.5, \mathrm{CHCl}) ;{ }^{1} \mathrm{H} \mathrm{NMR}(250$ $\left.\mathrm{MHz} \mathrm{CDCl}_{3}\right) \delta: 7.21(2 \mathrm{H}, \mathrm{d}, J=8.8 \mathrm{~Hz}), 6.81(2 \mathrm{H}, \mathrm{d}, J=8.8 \mathrm{~Hz}), 5.30(1 \mathrm{H} \mathrm{d}, J=9.5 \mathrm{~Hz}), 5.07(1 \mathrm{H}, \mathrm{d}, J=9.5 \mathrm{~Hz}), 4.35(1 \mathrm{H}, \mathrm{d}, J=1.8$ $\mathrm{Hz}), 3.74(3 \mathrm{H}, \mathrm{s}), 3.71(3 \mathrm{H}, \mathrm{s}), 3.16(1 \mathrm{H}, \mathrm{d}, J=4.3 \mathrm{~Hz}), 1.37(9 \mathrm{H}, \mathrm{s}) ;{ }^{13} \mathrm{C} \mathrm{NMR}\left(62.5 \mathrm{MHz}, \mathrm{CDCl}_{3}\right) \delta: 173.4,159.1,155.1,131.3$, $127.9,113.9,79.8,76.5,73.6,55.2,52.9,28.3$. 
(1R, 2R)-1-(tert-Butoxycarbonylamino)-3-methoxy-1-(4-methoxyphenyl)-3-oxopropan-2yl-4-nitrobenzoate (15a):

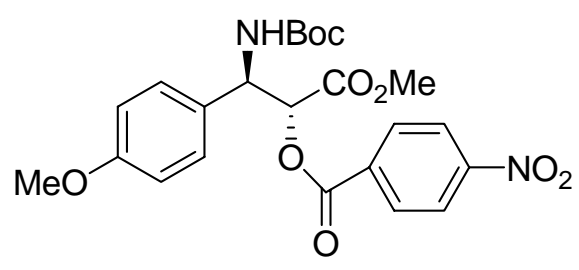

$15 a$

To a stirred solution of compound $15(0.5 \mathrm{~g}, 1.5 \mathrm{mmol})$ in anhydrous THF $(25 \mathrm{~mL})$ were added $\mathrm{PPh}_{3}(1.2 \mathrm{~g}$, $4.6 \mathrm{mmol})$ and 4 nitrobenzoic acid $(0.77 \mathrm{~g}, 4.6 \mathrm{mmol})$. The solution was cooled to $0{ }^{\circ} \mathrm{C}$ and DIAD $(932 \mu \mathrm{L}, 4.6 \mathrm{mmol})$ was slowly added. The reaction mixture was allowed to stir at room temperature for six hours under a nitrogen atmosphere. THF was removed by rotary evaporation and the crude product was partitioned between water and ethyl acetate. The combine organic extracts were washed with brine, dried over $\mathrm{Na}_{2} \mathrm{SO}_{4}$ and concentrated by rotary evaporation. The residue was purified by flash column chromatography to give compound 15a $(0.5 \mathrm{~g}, 70 \%)$ as a yellowish syrup. ${ }^{1} \mathrm{H} \mathrm{NMR}\left(250 \mathrm{MHz}, \mathrm{CDCl}_{3}\right): \delta 8.22(2 \mathrm{H}, \mathrm{d}, J=8.8 \mathrm{~Hz}), 8.10(2 \mathrm{H}, \mathrm{d}, J=8.8 \mathrm{~Hz}), 7.21(2 \mathrm{H}, \mathrm{d}$, $J=8.8 \mathrm{~Hz}), 6.82(2 \mathrm{H}, \mathrm{d}, J=8.8 \mathrm{~Hz}) .5 .58(1 \mathrm{H}, \mathrm{d}, J=4.3 \mathrm{~Hz}), 5.25(1 \mathrm{H}, \mathrm{br} \mathrm{s}), 5.23(1 \mathrm{H}, \mathrm{d}, J=7.0 \mathrm{~Hz}), 3.73(3 \mathrm{H}, \mathrm{s}), 3.61(3 \mathrm{H}, \mathrm{s}), 1.35$ $(9 \mathrm{H}, \mathrm{s}) ;{ }^{13} \mathrm{C} \mathrm{NMR}\left(62.5 \mathrm{MHz}, \mathrm{CDCl}_{3}\right): \delta 167.7,163.8,159.6,154.8,150.8,134.5,131.0,128.6,128.3,123.6,114.1,80.4,76.5,75.3$, $55.2,54.5,52.6,28.2$. 


\section{(2R, 3R)-Methyl 3-tert-butoxycarbonylamino-2-hydroxy-3-(4-methoxyphenyl)propanoate (16) :}

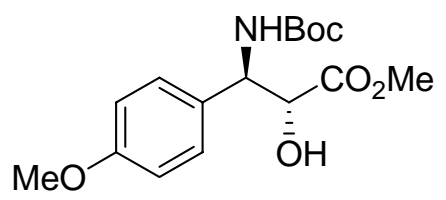

16

Triethylamine $(446 \mu \mathrm{L}, 3.1 \mathrm{mmol})$ was added dropwise to a stirred solution of compound $\mathbf{1 5 a}(0.5 \mathrm{~g}, 1.0 \mathrm{mmol})$ in anhydrous THF (20 $\mathrm{mL}$ ) under a nitrogen atmosphere. The reaction mixture was stirred at room temperature for one hour and concentrated to dryness by rotary evaporation. The crude product was partitioned between water and ethyl acetate then extracted with ethyl acetate, dried over $\mathrm{Na}_{2} \mathrm{SO}_{4}$ and concentrated by rotary evaporation. The crude product was purified by flash column chromatography to afford compound $16(0.33 \mathrm{~g}, 98 \%)$ as a pale yellow solid; mp $150-152{ }^{\circ} \mathrm{C} ;[\alpha]_{\mathrm{D}}{ }^{23}-1.8\left(\mathrm{c}=0.5, \mathrm{CHCl}_{3}\right) ;{ }^{1} \mathrm{H} \mathrm{NMR}\left(250 \mathrm{MHz}, \mathrm{CDCl}_{3}\right) \delta: 7.09(2 \mathrm{H}, \mathrm{d}, \mathrm{J}=$ $8.5 \mathrm{~Hz}), 6.76(2 \mathrm{H}, \mathrm{d}, J=8.8 \mathrm{~Hz}), 5.49(1 \mathrm{H}, \mathrm{d}, J=8.3 \mathrm{~Hz}), 4.97(1 \mathrm{H}, \mathrm{d}, J=6.3 \mathrm{~Hz}), 4.50(1 \mathrm{H}, \mathrm{dd}, J=3.3,6.5 \mathrm{~Hz}), 3.70(3 \mathrm{H}, \mathrm{s}), 3.65$ $(3 \mathrm{H}, \mathrm{s}), 2.85(1 \mathrm{H}, \mathrm{d}, J=6.5 \mathrm{~Hz}), 1.37(9 \mathrm{H}, \mathrm{s}) ;{ }^{13} \mathrm{C} \mathrm{NMR}\left(62.5 \mathrm{MHz}, \mathrm{CDCl}_{3}\right) \delta: 172.4,159.3,154.9,128.9,128.4,113.8,79.8,76.5$, $73.3,56.1,55.1,52.5,28.3$. 
tert-Butyl (1R, 2R)-2,3-dihydroxy-1-(4-methoxphenyl)propylcarbamate (17):

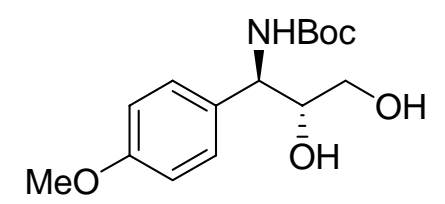

17

To an ice-cooled solution of $\mathrm{NaBH}_{4}(0.08 \mathrm{~g}, 2.3 \mathrm{mmol})$ in anhydrous methanol $(10 \mathrm{~mL})$ was added dropwise a solution of compound $16(0.25 \mathrm{~g}, 0.7 \mathrm{mmol})$ in anhydrous methanol $(5 \mathrm{~mL})$. The reaction mixture was stirred at room temperature for four hours and the solvent was removed by rotary evaporation. The crude product was partitioned between water and ethyl acetate then extracted with ethyl acetate, washed with saturated $\mathrm{NH}_{4} \mathrm{Cl}$ solution, brine, dried over $\mathrm{Na}_{2} \mathrm{SO}_{4}$ and concentrated by rotary evaporation. The crude product was purified by flash column chromatography to give compound $17(0.21 \mathrm{~g}, 92 \%)$ as a white solid; mp $116-118{ }^{\circ} \mathrm{C}(\mathrm{Lit}$, mp $\left.11{ }^{\circ} \mathrm{C}\right) ;[\alpha]_{\mathrm{D}}^{23}-50.2\left(\mathrm{c}=0.5 \mathrm{M}, \mathrm{CHCl}_{3}\right)\left[\mathrm{Lit},[\alpha]_{\mathrm{D}}{ }^{23}-51.2\left(\mathrm{c}=1 \mathrm{M}, \mathrm{CHCl}_{3}\right)\right] .{ }^{1} \mathrm{H} \mathrm{NMR}\left(250 \mathrm{MHz}, \mathrm{CDCl}_{3}\right) \delta: 7.17(2 \mathrm{H}, \mathrm{d}, J=8.8 \mathrm{~Hz})$, $6.82(2 \mathrm{H}, \mathrm{dd}, J=2.8,5.0 \mathrm{~Hz}), 5.18(1 \mathrm{H}, \mathrm{br} \mathrm{s}), 4.55(1 \mathrm{H}, \mathrm{m}), 3.76-3.70(1 \mathrm{H}, \mathrm{m}), 3.72(3 \mathrm{H}, \mathrm{s}), 3.59(2 \mathrm{H}, \mathrm{d}, J=2.5 \mathrm{~Hz}), 2.52(2 \mathrm{H}, \mathrm{d}, J=$ $2.5 \mathrm{~Hz}), 1.39(9 \mathrm{H}, \mathrm{s}) ;{ }^{13} \mathrm{C} \mathrm{NMR}(62.5 \mathrm{MHz}$, acetone-d 6 ) $\delta: 160.6,158.9,133.9,128.4,115.0,85.8,62.4,57.6,55.6,28.9$. 


\section{(4R, 5R)-5-(Hydroxymethyl)-4-(4-methoxyphenyl)oxazolidin-2one (9):}

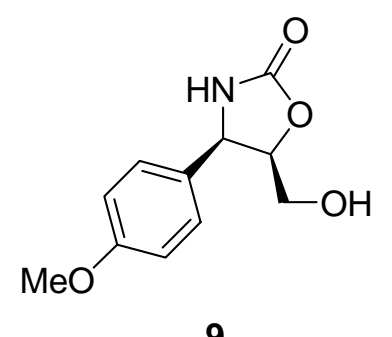

9

To a solution of compound $17(0.1 \mathrm{~g}, 0.3 \mathrm{mmol})$ in anhydrous THF $(10 \mathrm{~mL})$ was added sodium hydride [0.04 g, $1.0 \mathrm{mmol}(60 \% \mathrm{w} / \mathrm{w}$ in mineral)] at room temperature and the mixture was stirred under a nitrogen atmosphere for two hours. The reaction mixture was concentrated, $\mathrm{CH}_{2} \mathrm{Cl}_{2}$ was added, washed with aqueous saturated $\mathrm{NH}_{4} \mathrm{Cl}$ solution, brine and dried over $\mathrm{Na}_{2} \mathrm{SO}_{4}$. The organic layer was concentrated by rotary evaporation and the residue was purified by flash column chromatography to give compound 9 ( $0.07 \mathrm{~g}, 92 \%)$ as a white solid; $\mathrm{mp} 117-118{ }^{\circ} \mathrm{C}$; $[\alpha]_{\mathrm{D}}{ }^{23}-68.2(\mathrm{c}=0.5 \mathrm{M}, \mathrm{MeOH})\left[\mathrm{Lit}:[\alpha]_{\mathrm{D}}{ }^{23}-69.7(\mathrm{c}=0.5 \mathrm{M}\right.$ in $\left.\mathrm{MeOH})\right]$; ${ }^{1} \mathrm{H} \mathrm{NMR}(250 \mathrm{MHz}$, acetone$\left.\mathrm{d}_{6}\right) \delta: 7.26(2 \mathrm{H}, \mathrm{d}, J=8.8 \mathrm{~Hz}), 6.96(2 \mathrm{H}, \mathrm{dd}, J=2.8,6.8 \mathrm{~Hz}), 5.04(1 \mathrm{H}, \mathrm{d}, J=8.3 \mathrm{~Hz}), 4.83(1 \mathrm{H}, \mathrm{ddd}, J=4.4,7.3,8.1 \mathrm{~Hz}), 3.87-3.80(1 \mathrm{H}$, m), $3.82(3 \mathrm{H}, \mathrm{s}), 3.19-3.26(2 \mathrm{H}, \mathrm{m}), 2.88(1 \mathrm{H}, \mathrm{s}) ;{ }^{13} \mathrm{C} \mathrm{NMR}\left(62.5 \mathrm{MHz}\right.$, acetone-d $\left.\mathrm{d}_{6}\right) \delta: 160.6,159.5,130.2,129.0,114.6,81.5,62.6$, 57.8, 55.5. 
(4R, 5S)-Methyl 4-(4-methoxyphenyl)-3-((R)-1-(4-methoxyphenyl)ethyl)-2-oxazolidine-5-carboxylate (18):

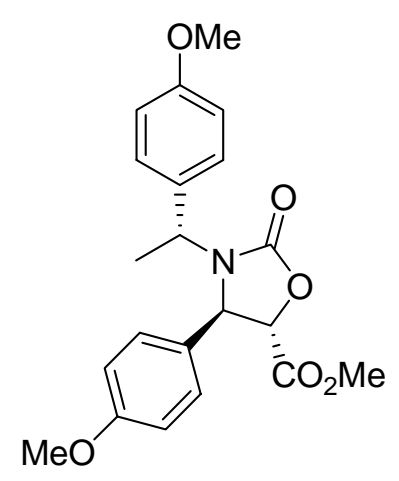

18

Compound 18 was synthesized from compound 14 employing a similar procedure as described for the synthesis of compound $\mathbf{3}$. mp $140-142{ }^{\circ} \mathrm{C} ;[\alpha]_{\mathrm{D}}{ }^{23}+42.8\left(\mathrm{c}=0.5, \mathrm{CHCl}_{3}\right) ;{ }^{1} \mathrm{H}$ NMR $\left(250 \mathrm{MHz}, \mathrm{CDCl}_{3}\right) \delta: 7.04-7.00(4 \mathrm{H}, \mathrm{m}), 6.82-6.76(4 \mathrm{H}, \mathrm{m}), 5.10(1 \mathrm{H}, \mathrm{q}, J=8.7$ $\mathrm{Hz}), 4.51(1 \mathrm{H}, \mathrm{d}, J=4.3 \mathrm{~Hz}), 4.14(1 \mathrm{H}, \mathrm{d}, J=4.3 \mathrm{~Hz}), 3.73(6 \mathrm{H}, \mathrm{s}), 3.61(3 \mathrm{H}, \mathrm{s}), 1.07(3 \mathrm{H}, \mathrm{d}, J=7.3 \mathrm{~Hz}) ;{ }^{13} \mathrm{C} \mathrm{NMR}(62.5 \mathrm{MHz}, \mathrm{CDCl})_{3}$ $\delta: 168.9,160.2,159.2,156.5,131.4,130.6,128.7,128.2,114.4,113.8,78.3,60.4,55.3,55.2,52.7,52.6,17.9$. 
(4R, 5S)-Methyl 4-(4-methoxyphenyl)oxazolidin-2-one-5-carboxylate (19):

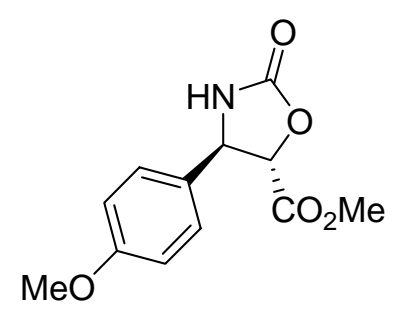

19

Compound 19 was synthesized from compound 18 employing a similar procedure as described for the synthesis of compound 4 . mp $88-90^{\circ} \mathrm{C} ;[\alpha]_{\mathrm{D}}{ }^{23}+87.4(\mathrm{c}=0.5, \mathrm{MeOH}) ;{ }^{1} \mathrm{H} \mathrm{NMR}\left(250 \mathrm{MHz}, \mathrm{CDCl}_{3}\right) \delta: 7.26(2 \mathrm{H}, \mathrm{d}, J=8.3 \mathrm{~Hz}), 6.88(2 \mathrm{H}, \mathrm{d}, J=8.3 \mathrm{~Hz}), 6.50(1 \mathrm{H}, \mathrm{s})$, $4.85(1 \mathrm{H}, \mathrm{d}, J=4.3 \mathrm{~Hz}), 4.65(1 \mathrm{H}, \mathrm{d}, J=4.3 \mathrm{~Hz}), 3.82(3 \mathrm{H}, \mathrm{s}), 3.72(3 \mathrm{H}, \mathrm{s}) .{ }^{13} \mathrm{C}-\mathrm{NMR}\left(250 \mathrm{MHz}, \mathrm{CDCl}_{3}\right) \delta: 167.8,159.1,157.0,129.8$, 126.2, 113.6, 79.4, 57.7, 54.3, 52.0. 
(4R, 5S)-5-(Hydroxymethyl)-4-(4-methoxyphenyl)oxazolidin-2-one (10):

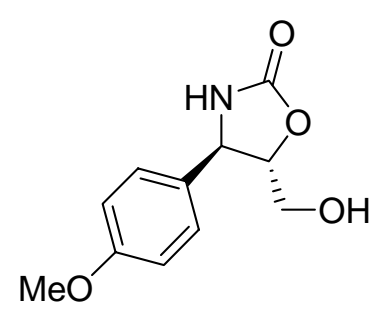

10

Compound 10 was synthesized from compound 19 employing the similar procedure as described for the synthesis of compound $\mathbf{5}$. mp $159-160{ }^{\circ} \mathrm{C} ;[\alpha]_{\mathrm{D}}{ }^{23}+30(\mathrm{c}=0.5, \mathrm{MeOH}) ;{ }^{1} \mathrm{H} \mathrm{NMR}\left(250 \mathrm{MHz}, \mathrm{CD}_{3} \mathrm{OH}\right) \delta: 7.20(2 \mathrm{H}, \mathrm{d}, J=8.5 \mathrm{~Hz}), 6.80(2 \mathrm{H}, \mathrm{d}, J=8.5 \mathrm{~Hz}), 4.65(1 \mathrm{H}, \mathrm{d}$, $J=6.5 \mathrm{~Hz}), 4.09-4.20(2 \mathrm{H}, \mathrm{m}), 3.78-3.52(2 \mathrm{H}, \mathrm{m}), 3.75(3 \mathrm{H}, \mathrm{s}), 2.72(1 \mathrm{H}, \mathrm{s}) ;{ }^{13} \mathrm{C} \mathrm{NMR}\left(250 \mathrm{MHz}, \mathrm{CD}_{3} \mathrm{OH}\right) \delta: 161.5,161.3,133.6$, 128.6, 115.4, 86.8, 62.5, 58.5, 55.8. 
${ }^{1}$ H NMR and ${ }^{13} \mathrm{C}$ NMR Spectra 


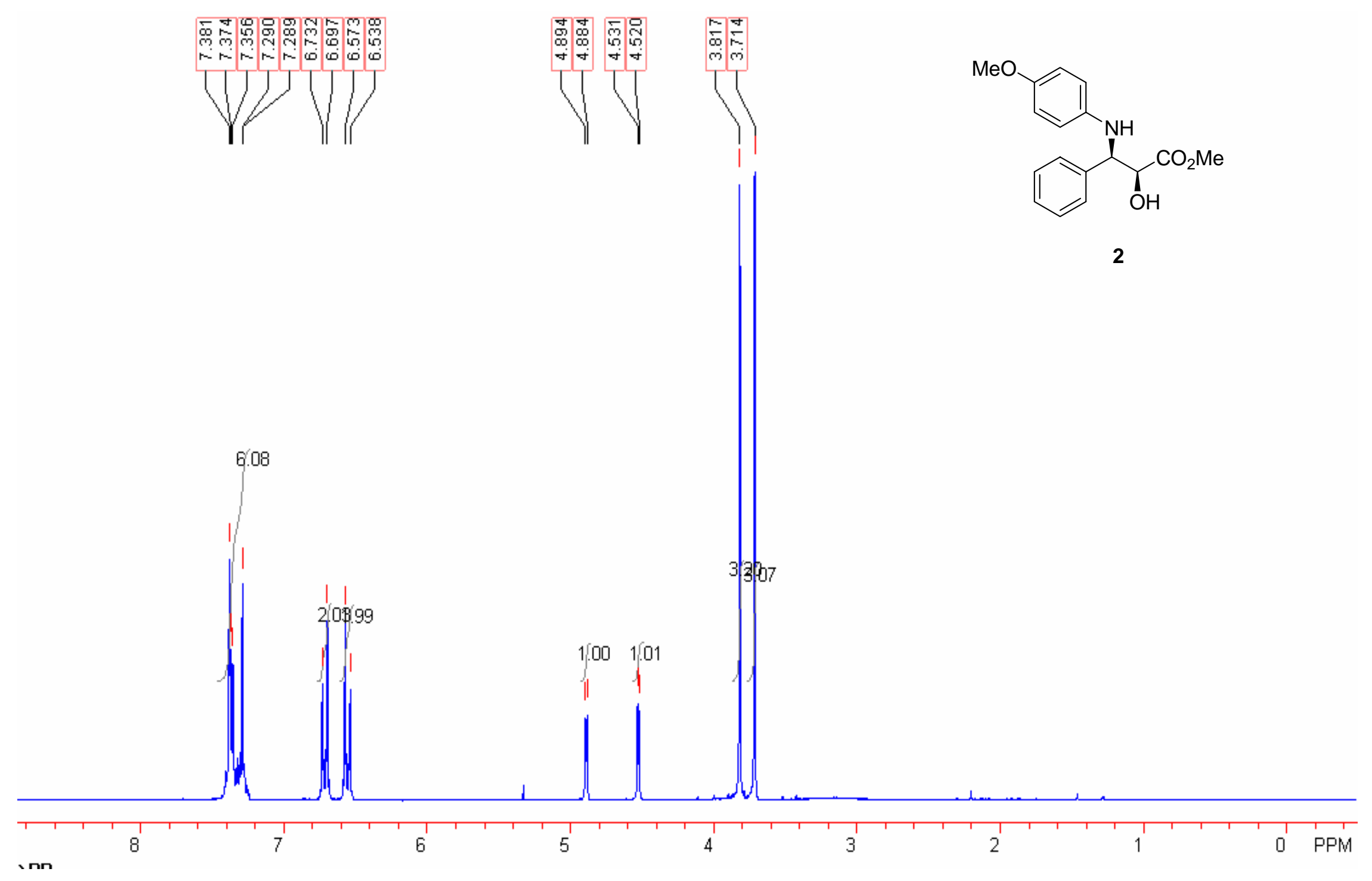




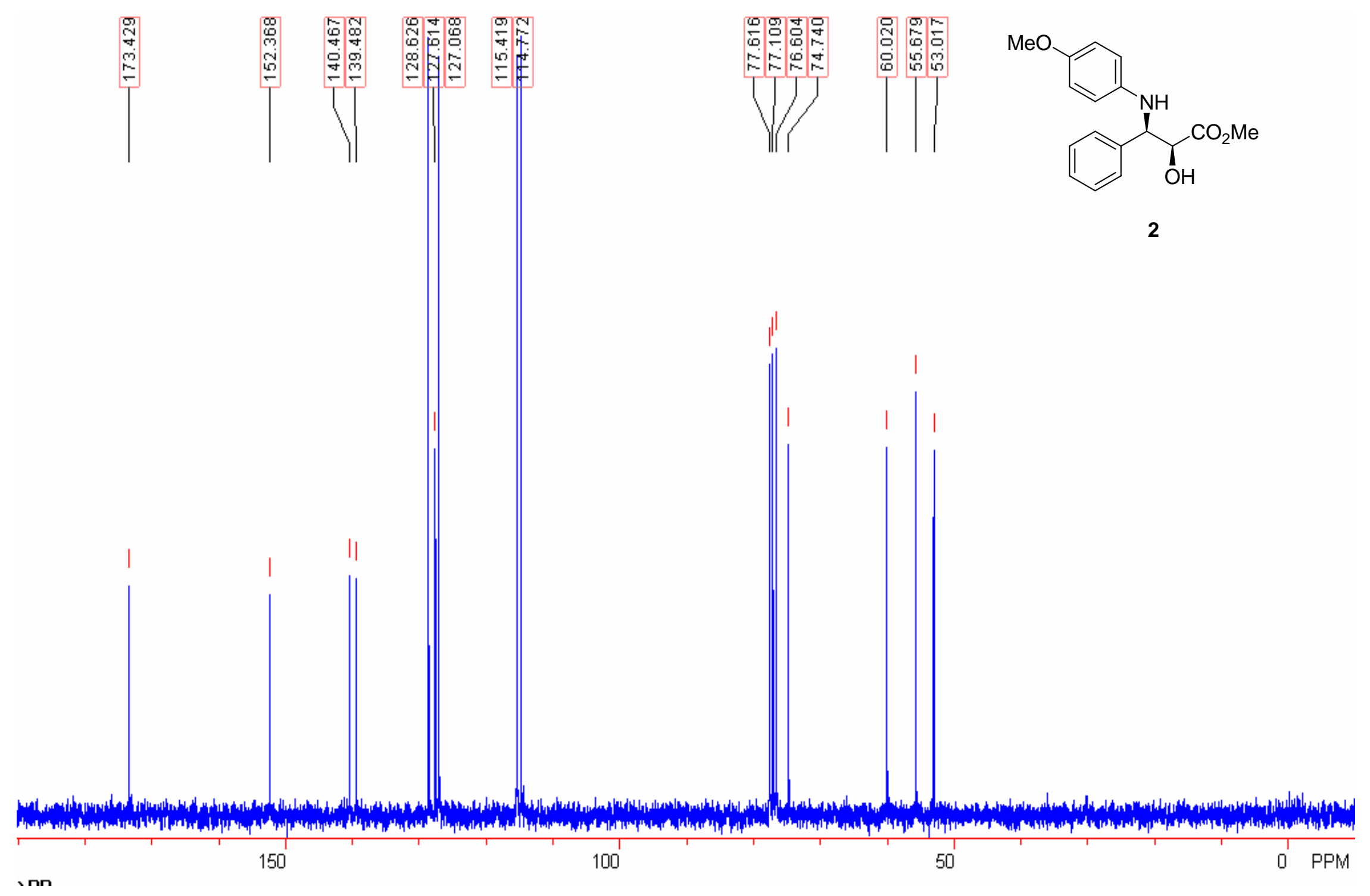




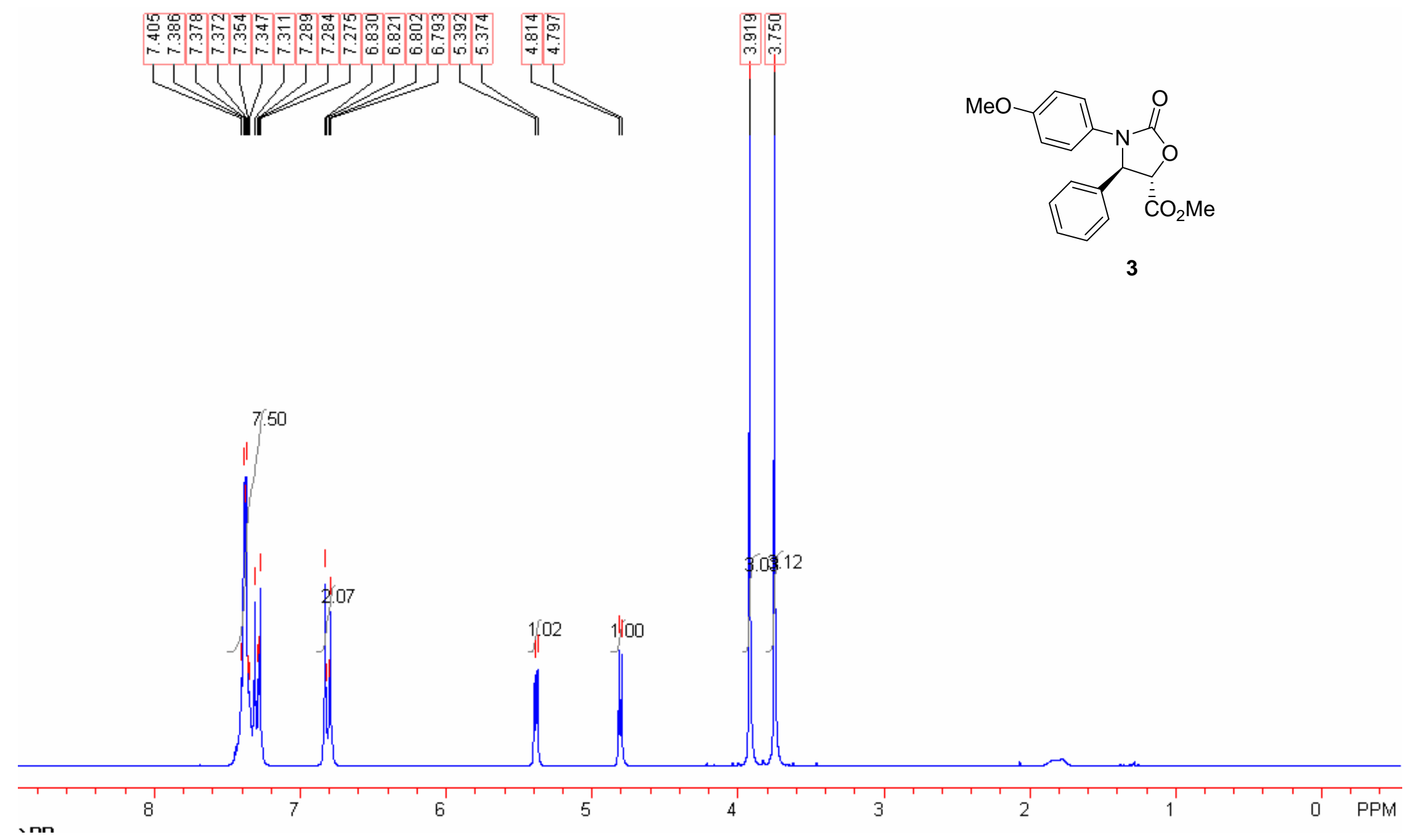




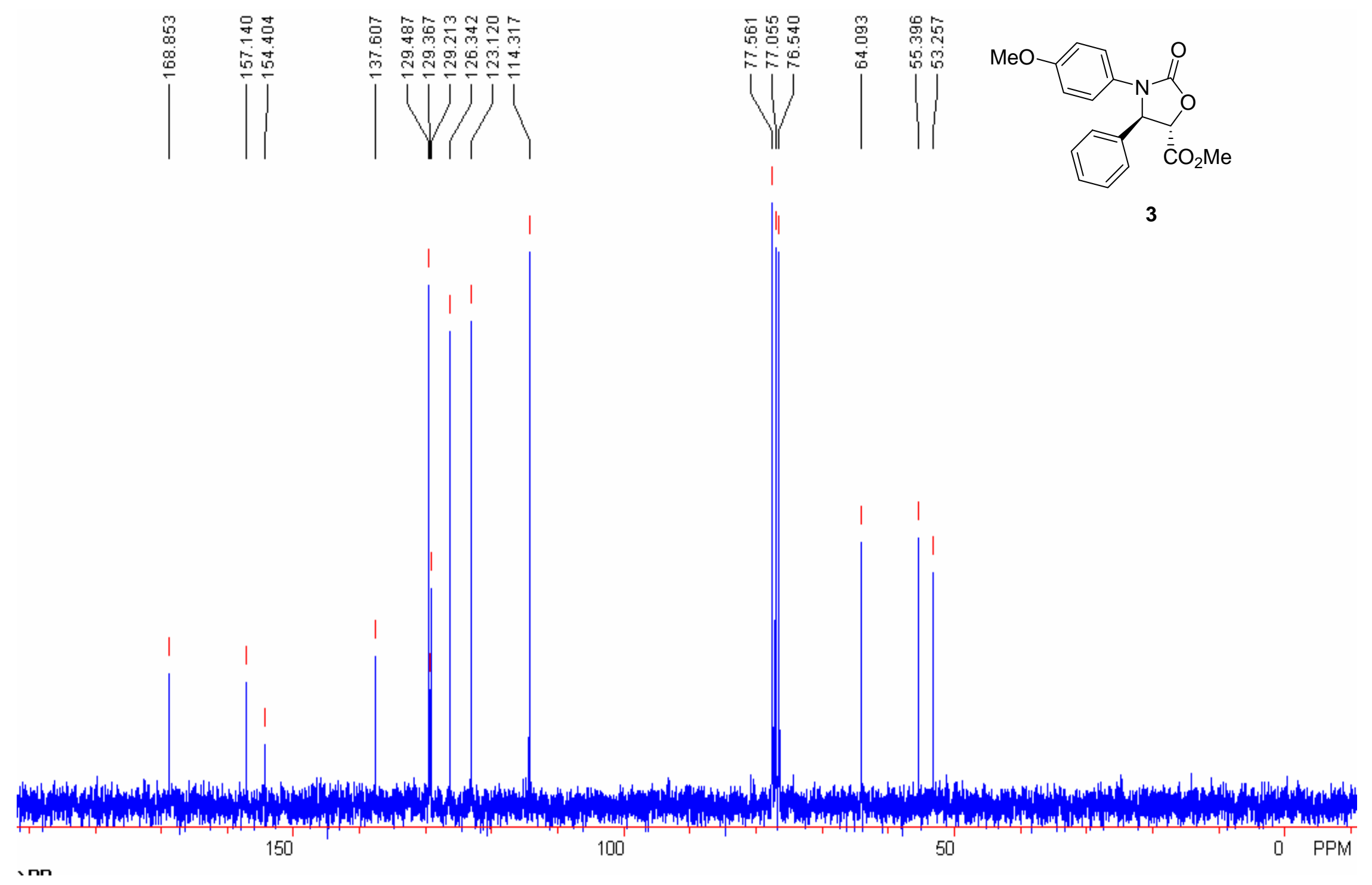




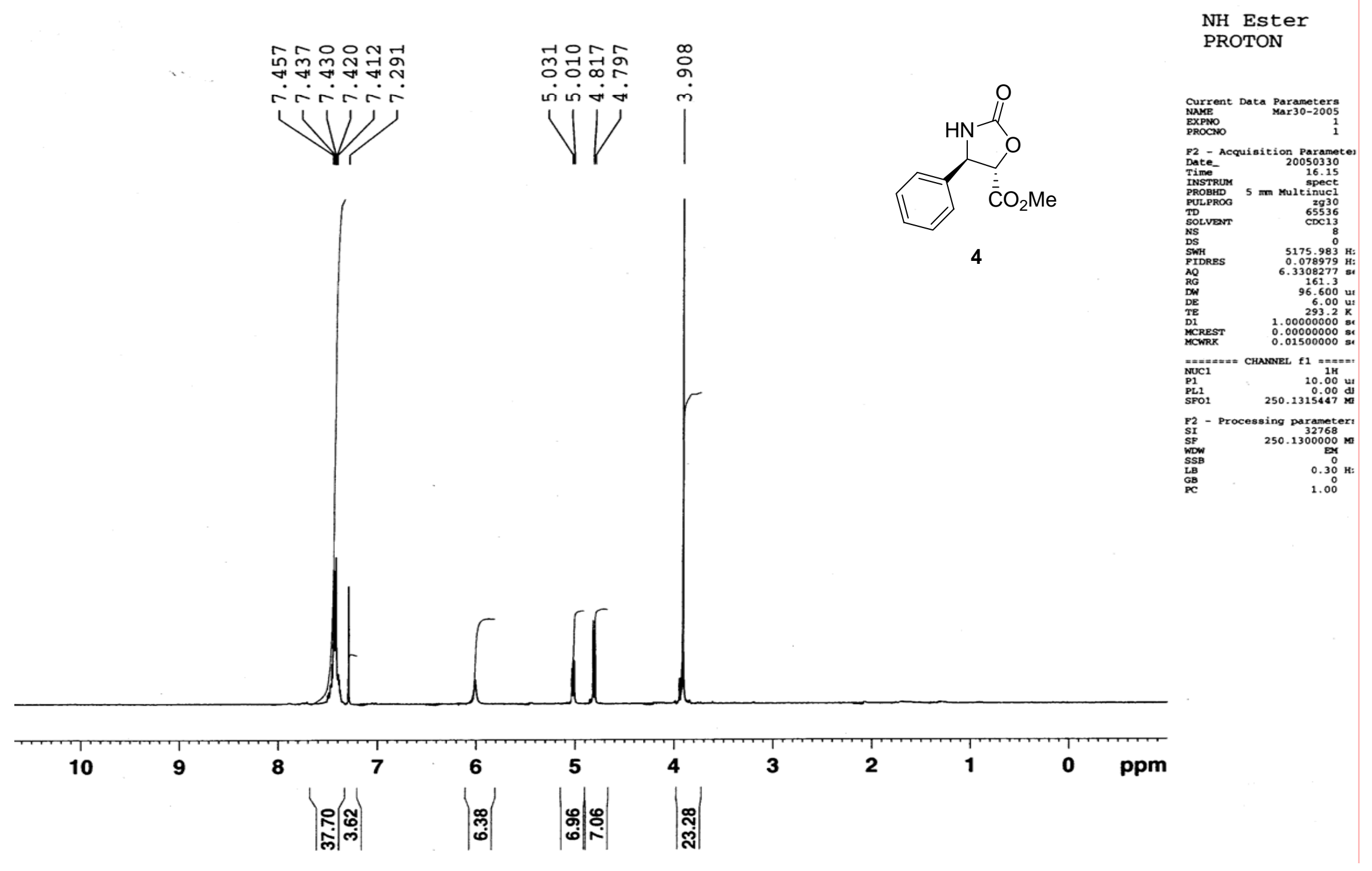



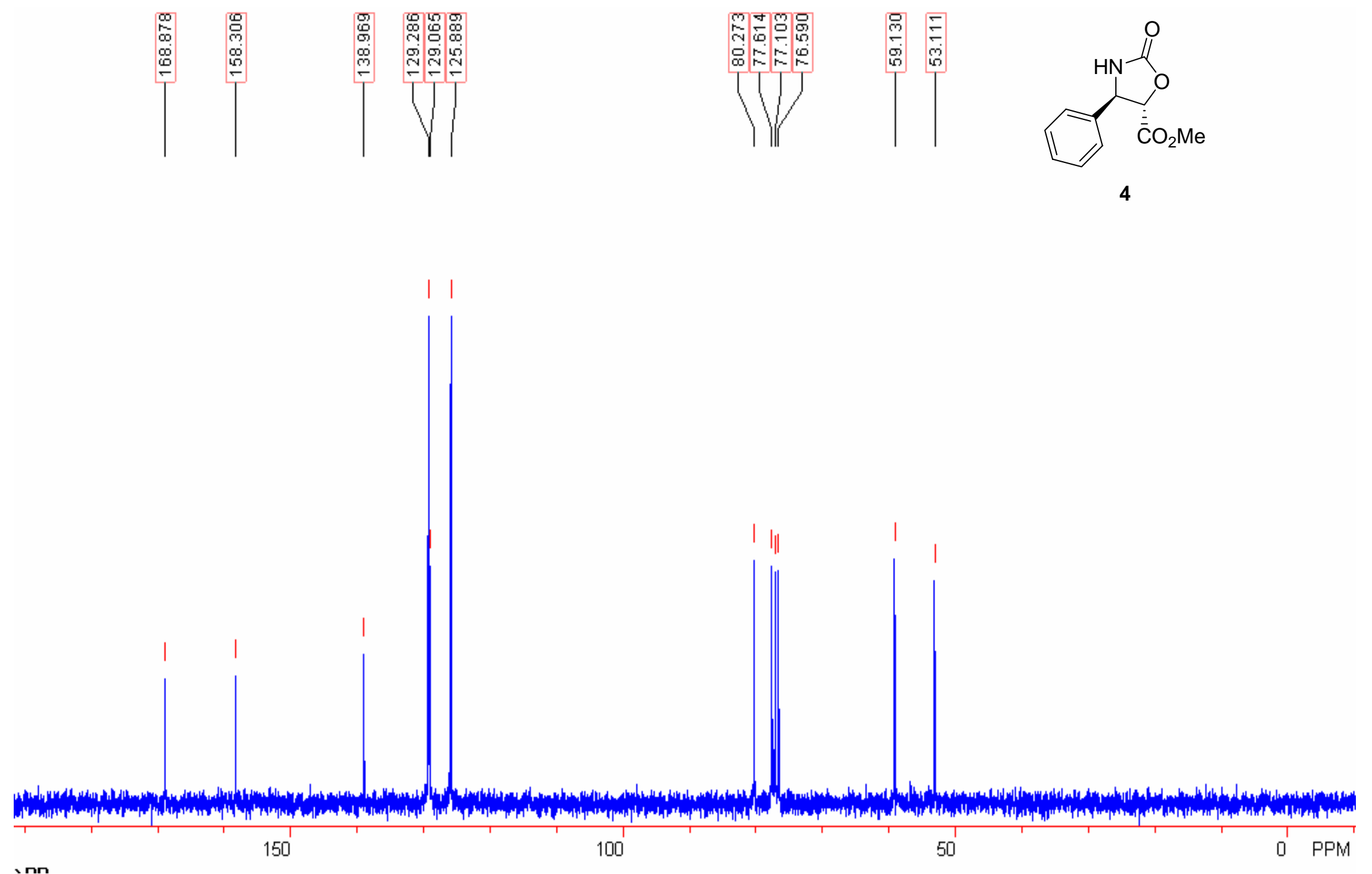


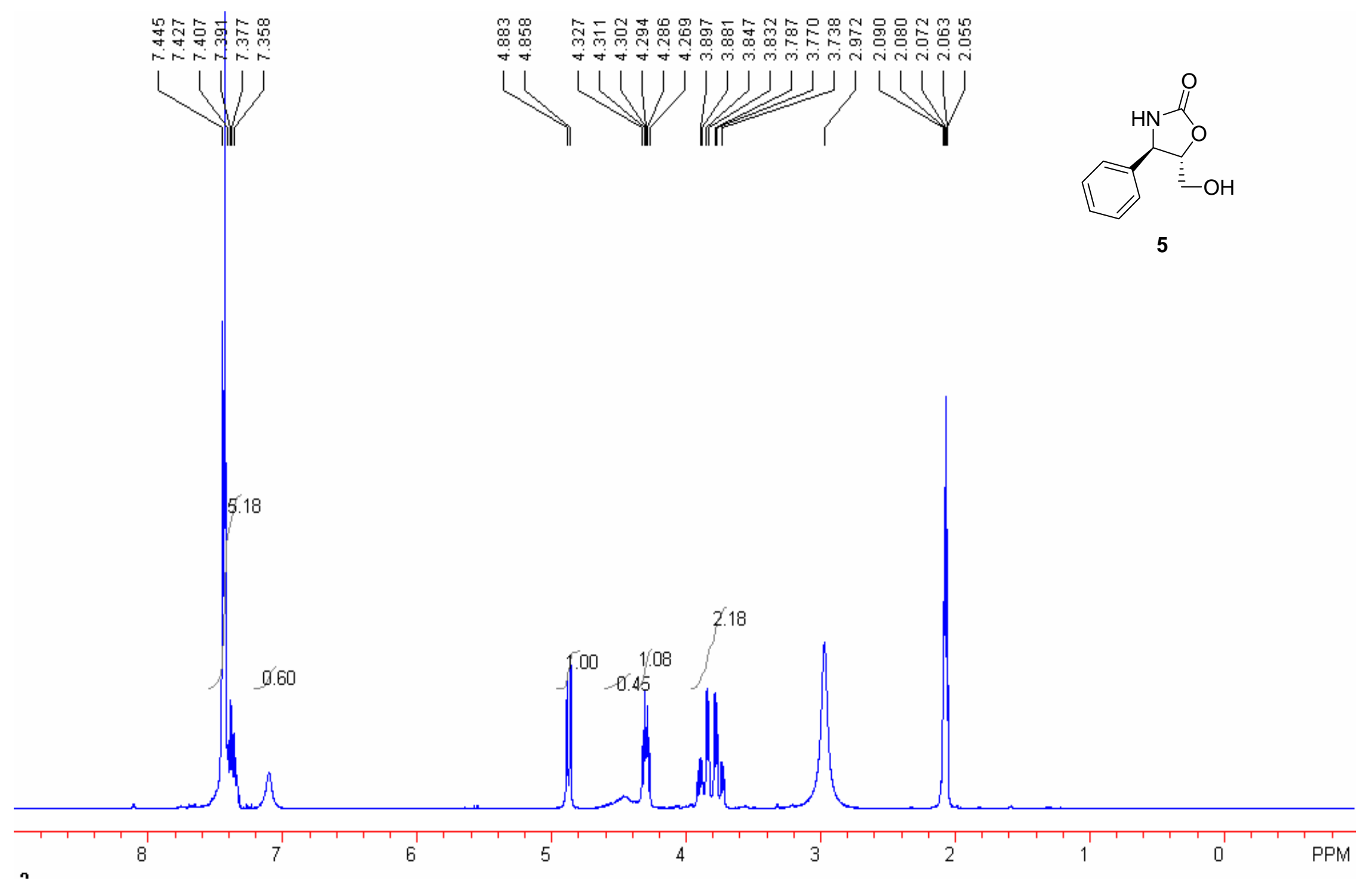




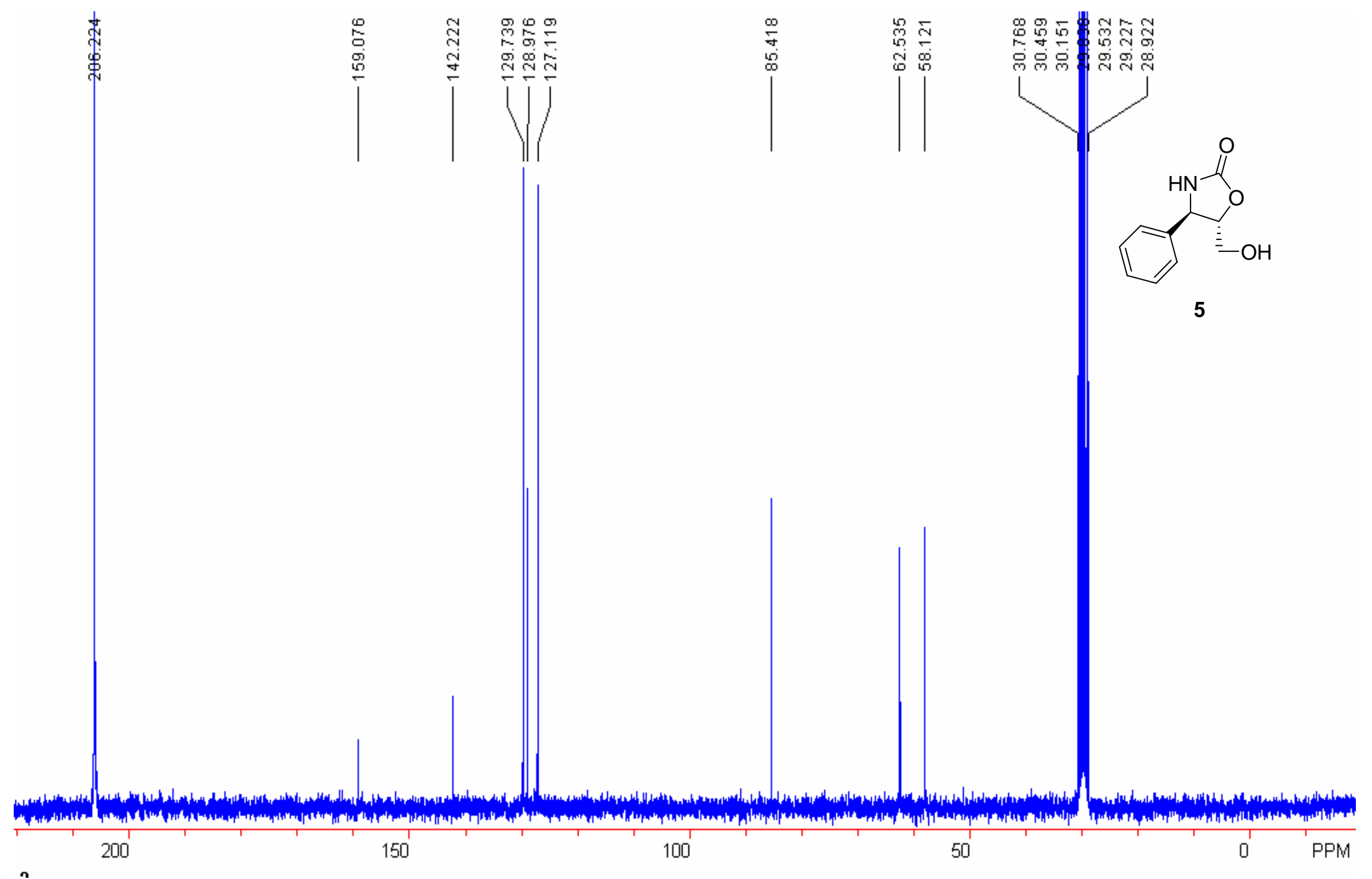




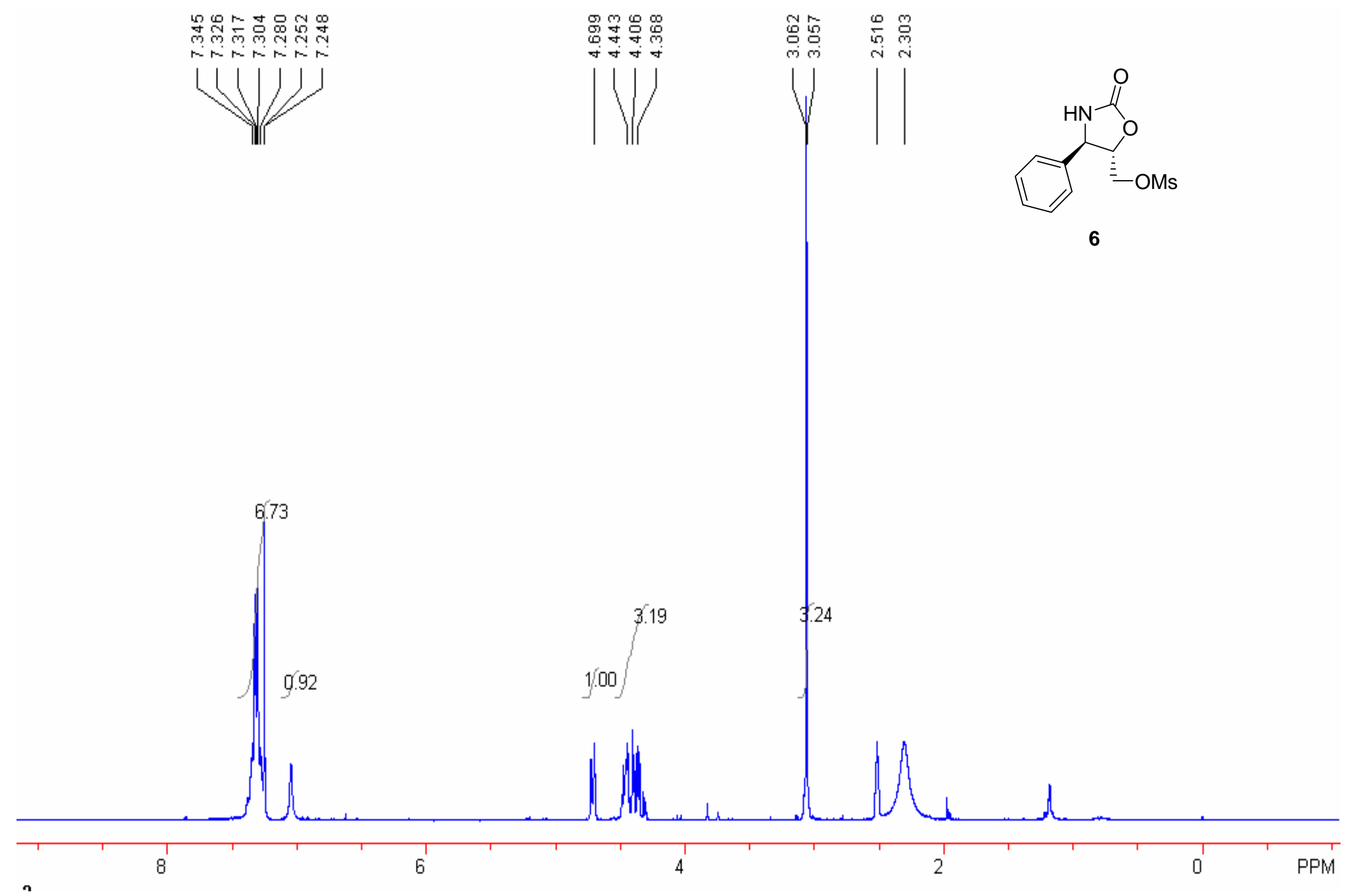



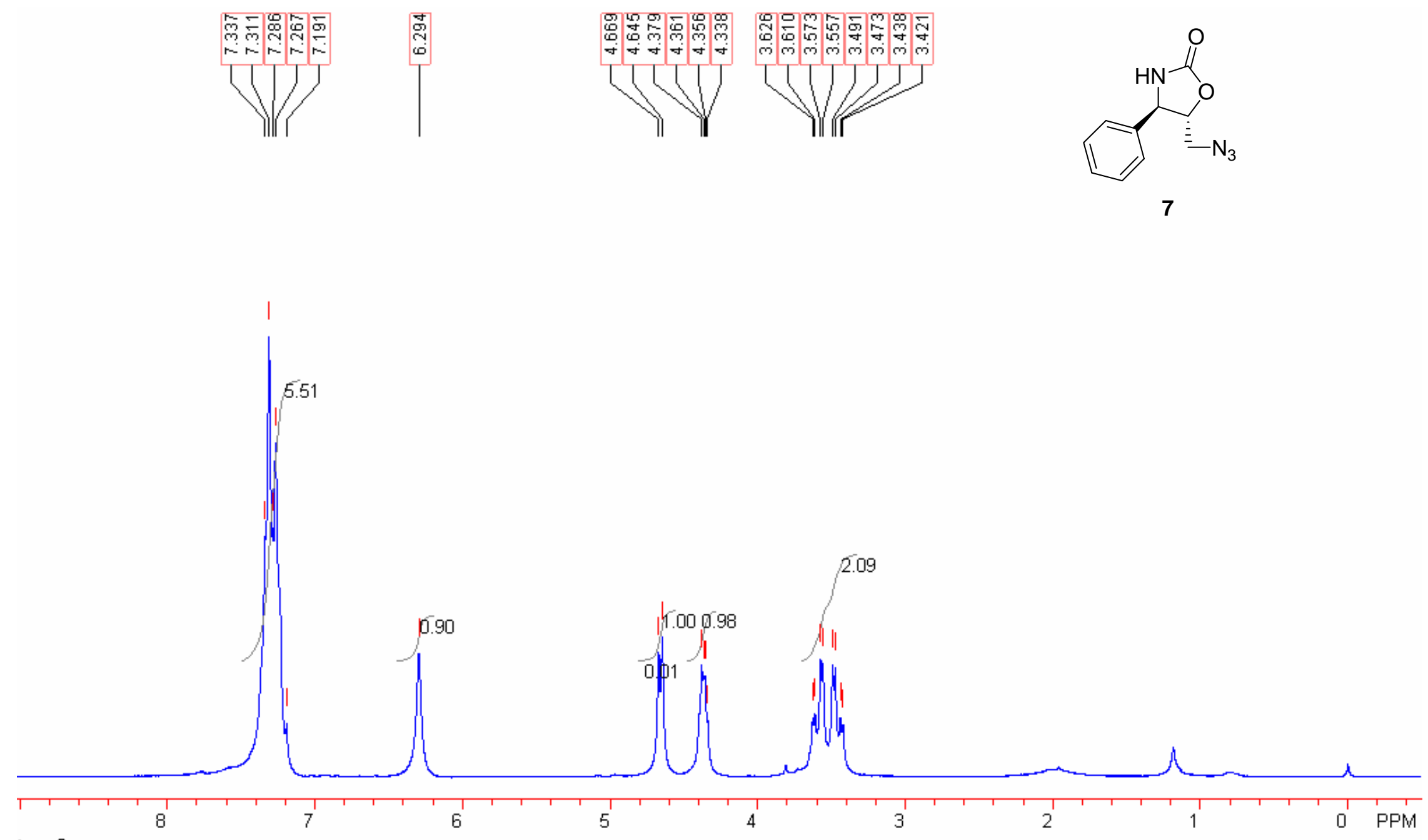

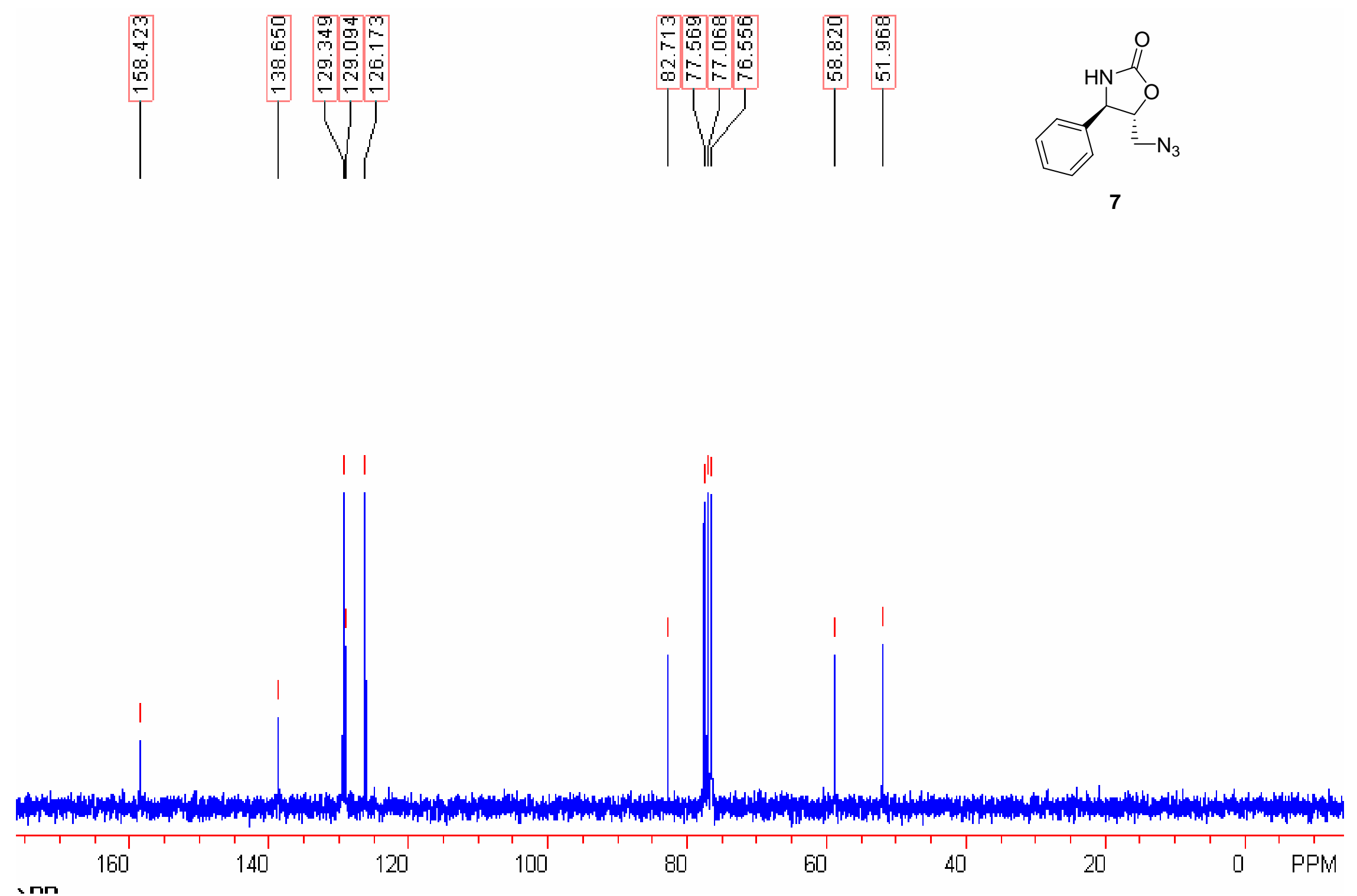

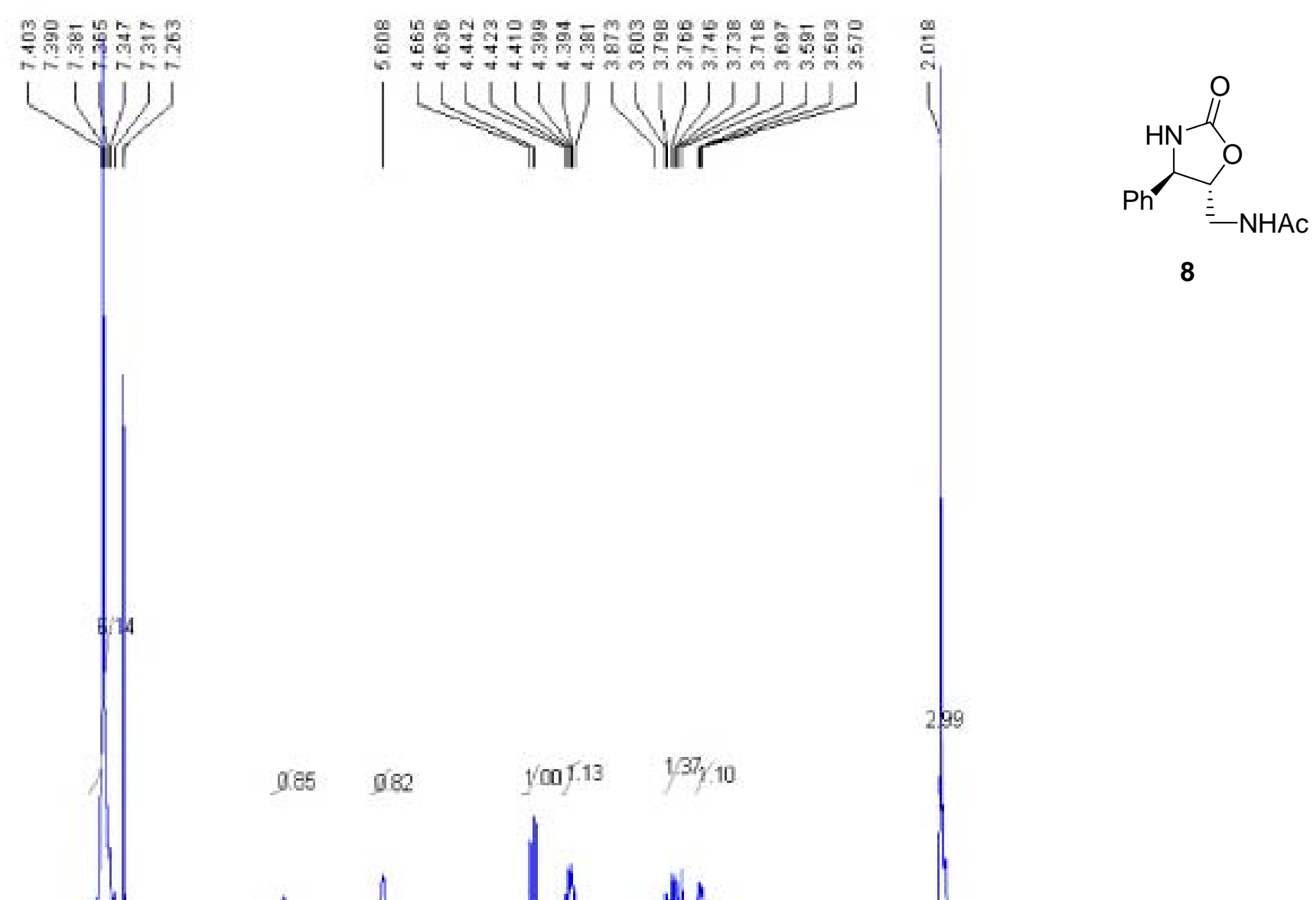

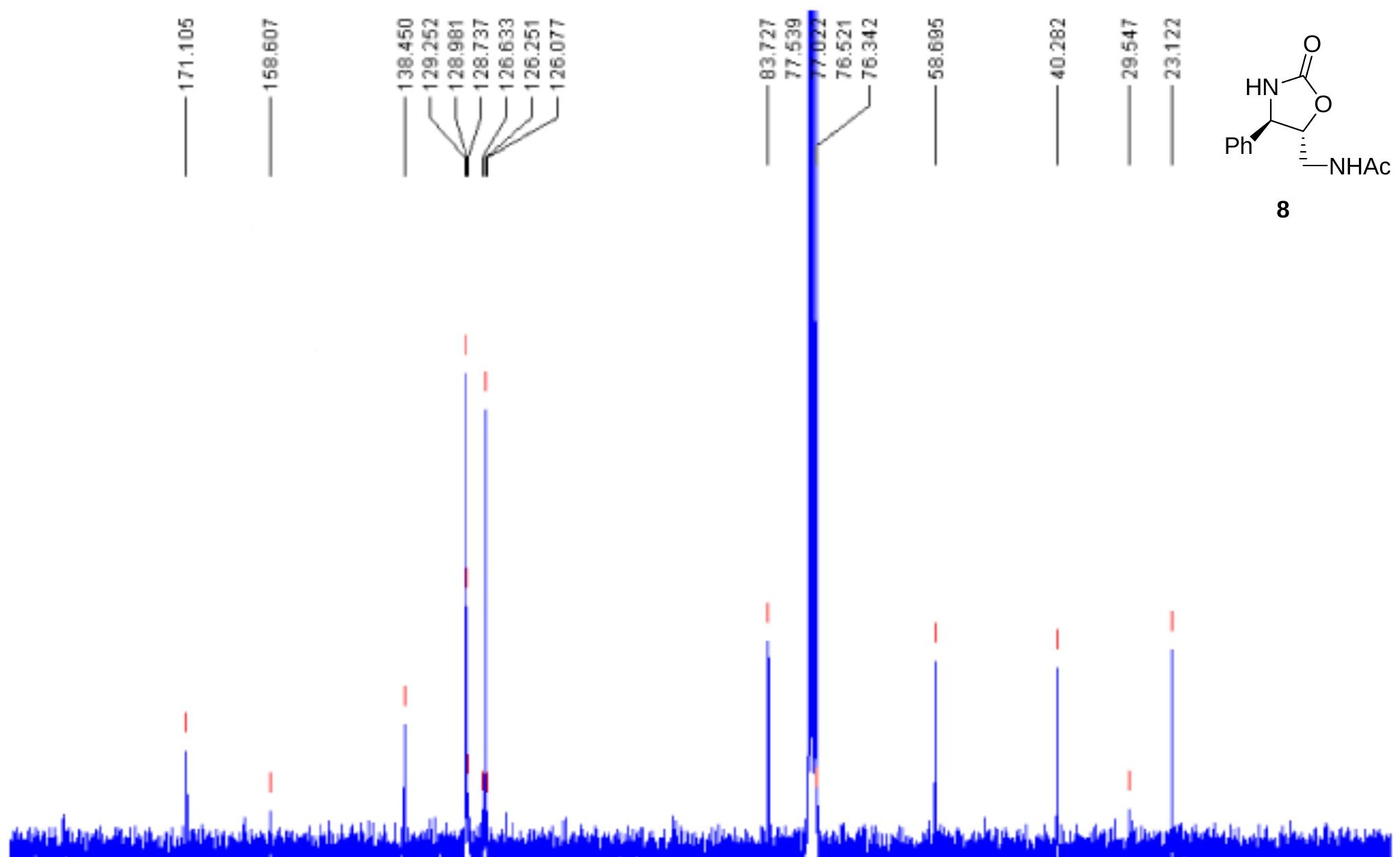


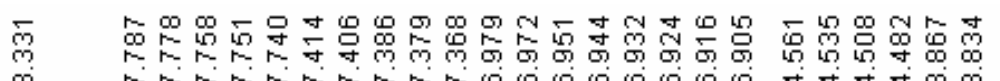

Q

111

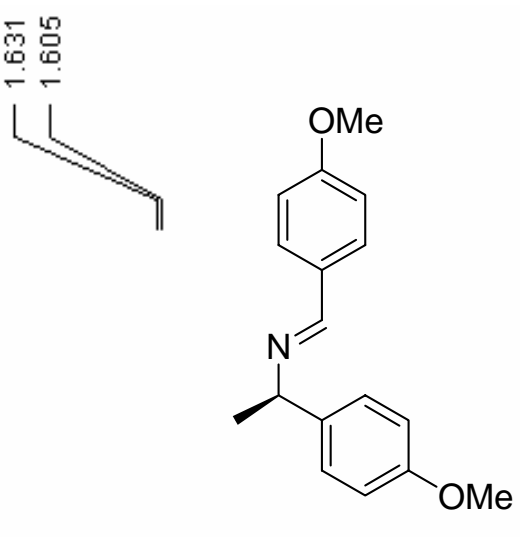

21

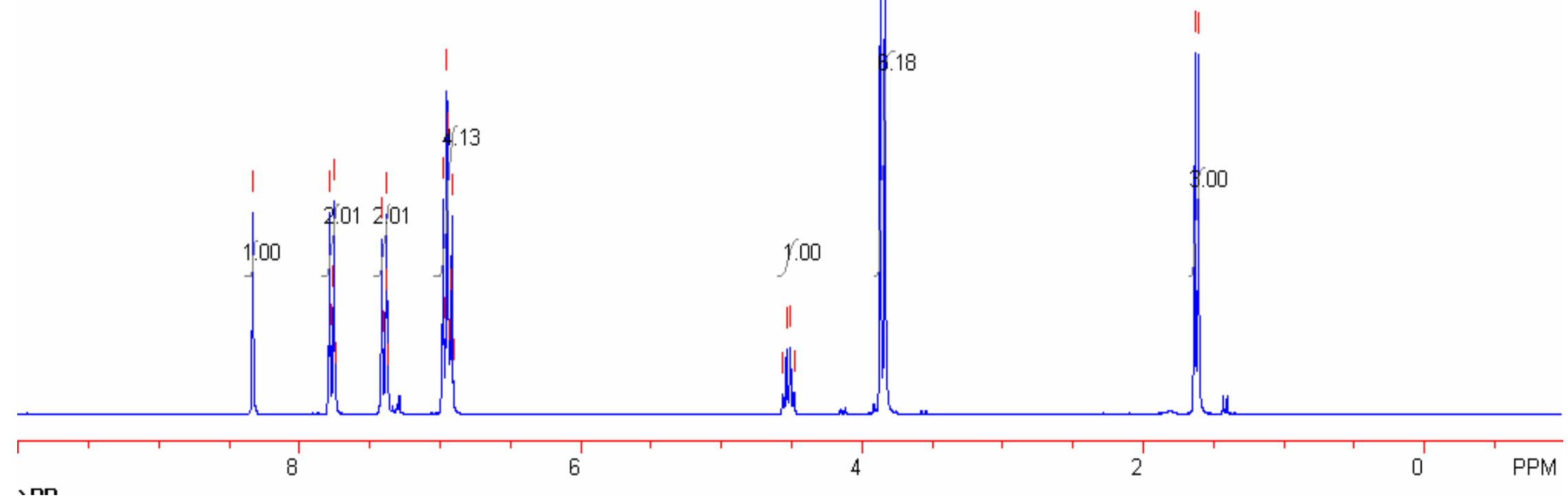




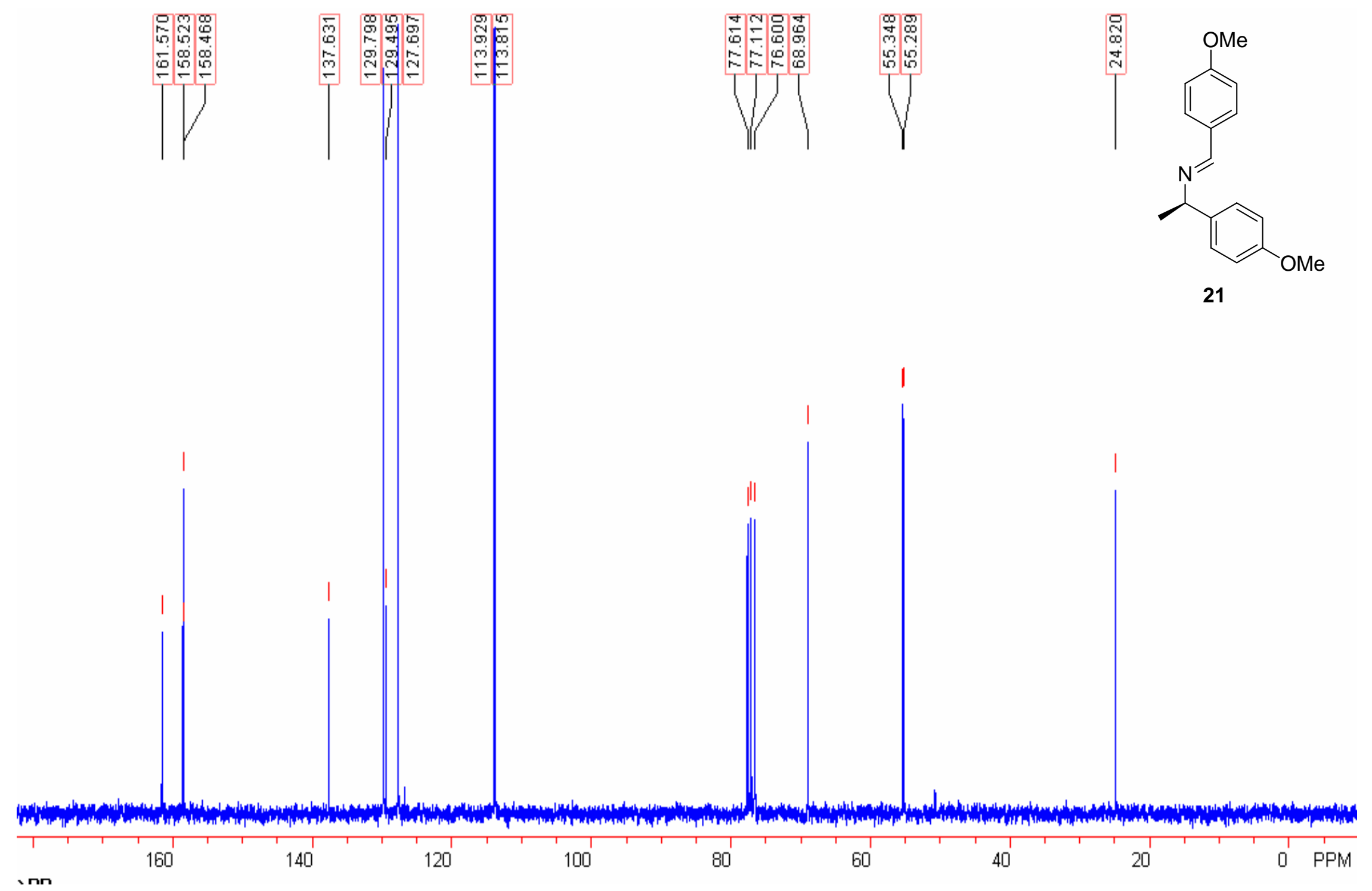




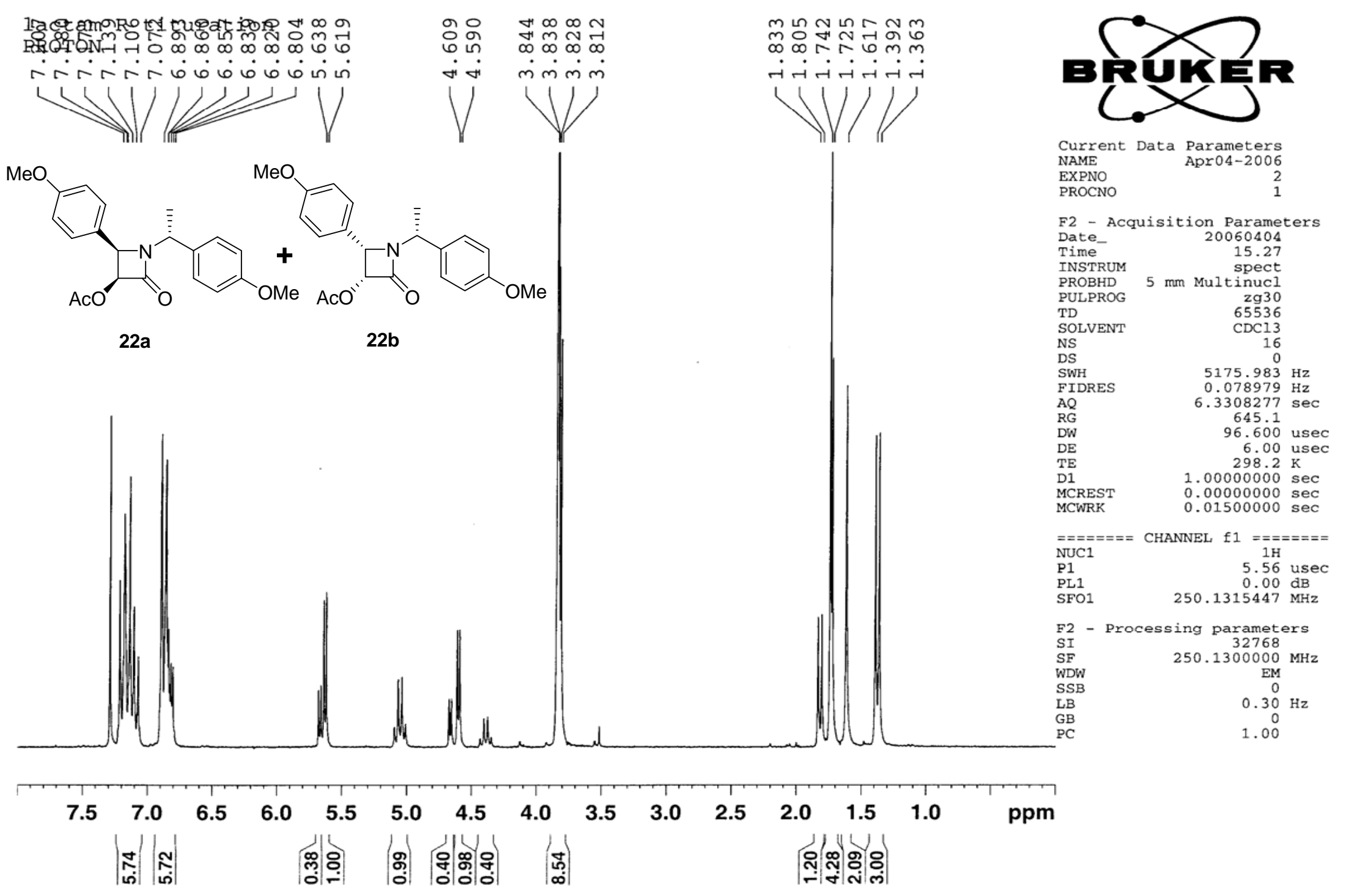


Lactam Chiral

C13CPD

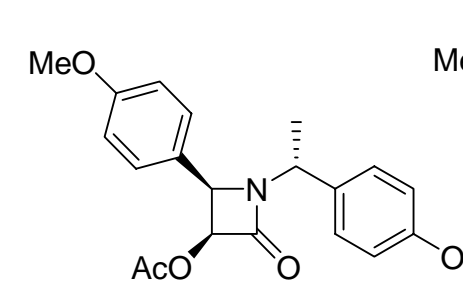

$22 a$
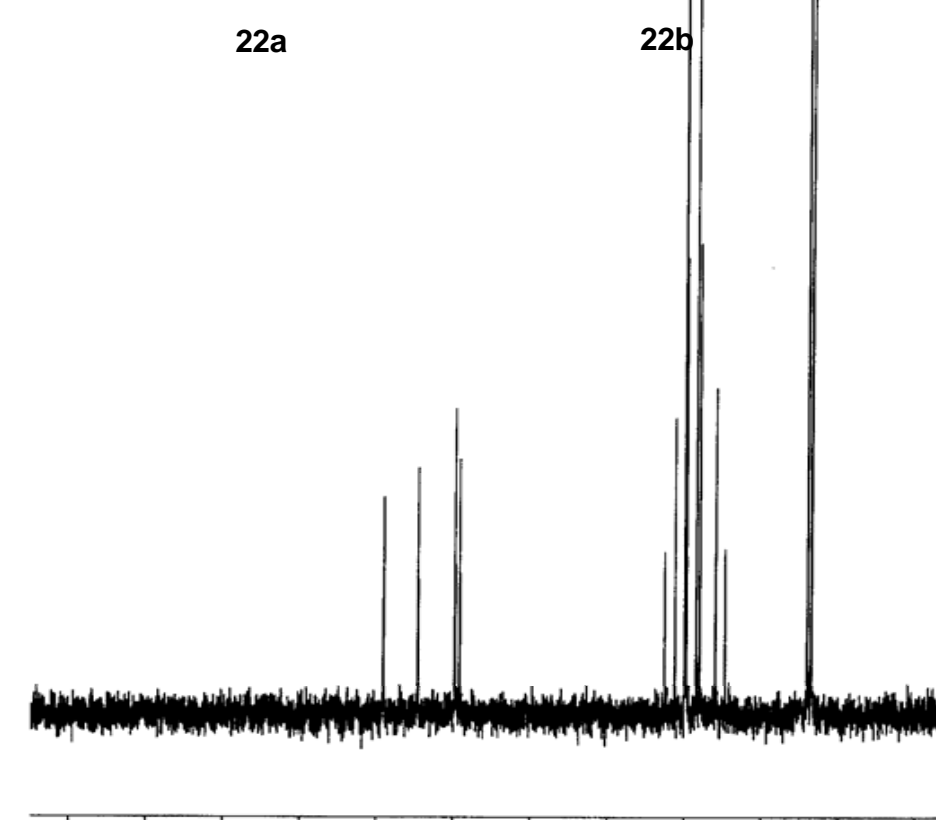

$4(4)(4)$

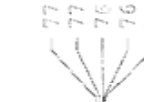

200

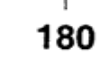

160
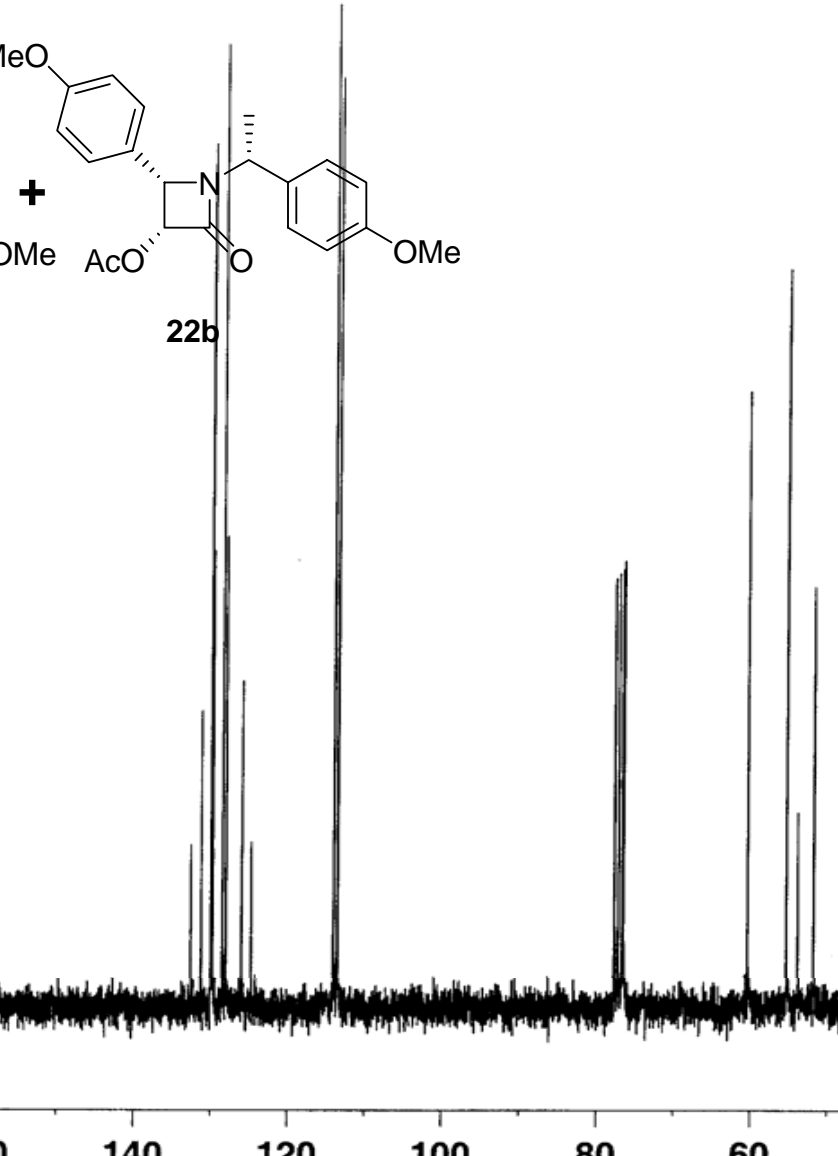

60
120
100
80

40

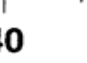

20

ppm

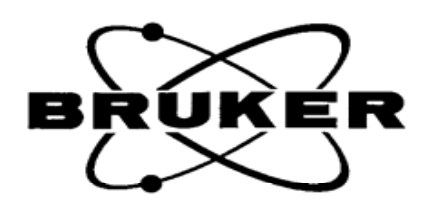

Current Data Parameters
NAME EXPNO PROCNO

F2 - Acquisition Parameters Time-

INSTRUM

PROBHD

TD

NS

$\begin{array}{lr}\text { DS } & 0 \\ \text { SWH } & 15060.241 \mathrm{~Hz} \\ \text { FIDRES } & 0.229801 \mathrm{~Hz}\end{array}$

$\mathrm{AQ}$
$\mathrm{RG}$

DW

TE

D11

DELTA

MCWRK

20060510
23.28

spect

tinucl

65536

$\mathrm{CDCl3}$
400

$15060.241 \mathrm{~Hz}$
$0.229801 \mathrm{~Hz}$ $2.1758451 \mathrm{sec}$

33.200 usec

6.00 usec

$298.2 \mathrm{~K}$

.0000000 sec

$1.89999998 \mathrm{sec}$

$.00000000 \mathrm{sec}$

$=======$ CHANNEL f $1 \quad=======$

${ }_{\mathrm{P} 1}^{\text {NUC1 }}$ 5.50 usec

$\begin{array}{lr}\text { PL1 } & -6.00 \mathrm{~dB} \\ \text { SFO1 } & 62.9015280 \mathrm{MHz}\end{array}$

$==x=====$ CHANNEL f2 $==x====$ = CPDPRG2 waltz16

$\begin{array}{ll}\text { NUC2 } & 100.00 \text { usec } \\ \text { PCPD2 } & 100.00 \text { as }\end{array}$

PL12

PL13

$0.00 \mathrm{~dB}$

$25.10 \mathrm{~dB}$

$250.1310005 \mathrm{MHz}$

F2 - Processing parameters

32768
$62.8952390 \mathrm{MHz}$

EM

0
$1.00 \mathrm{~Hz}$

1.40 


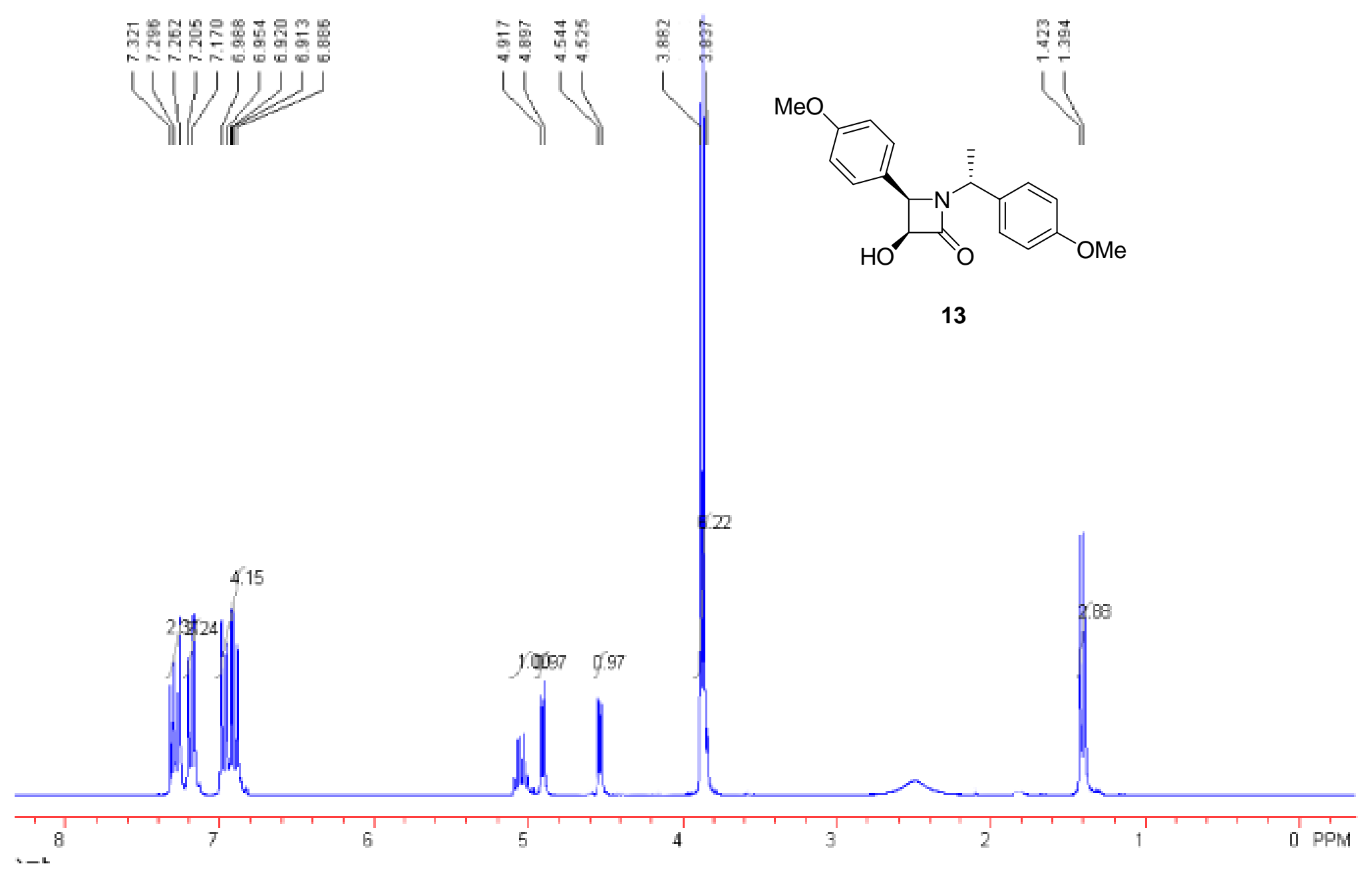



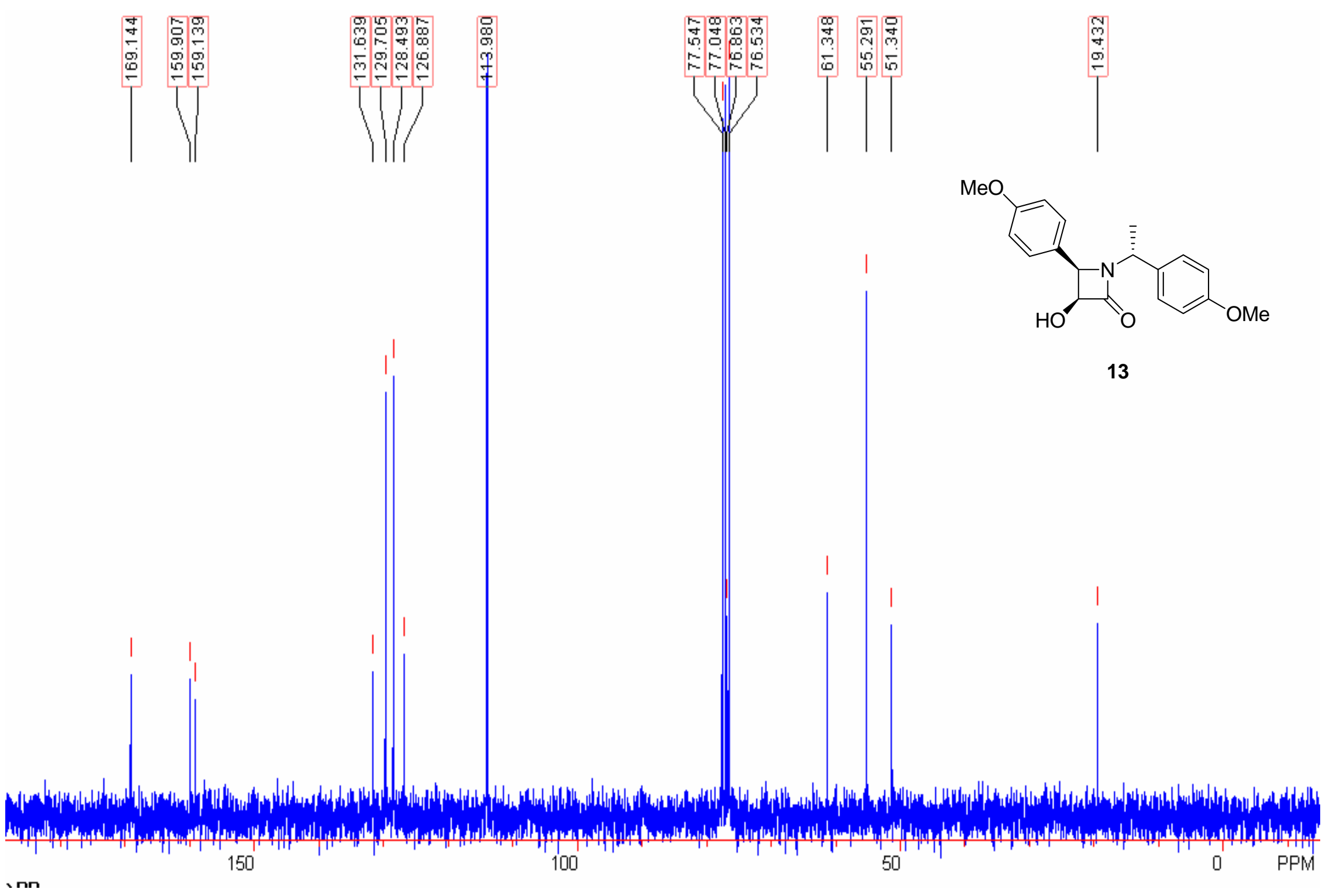


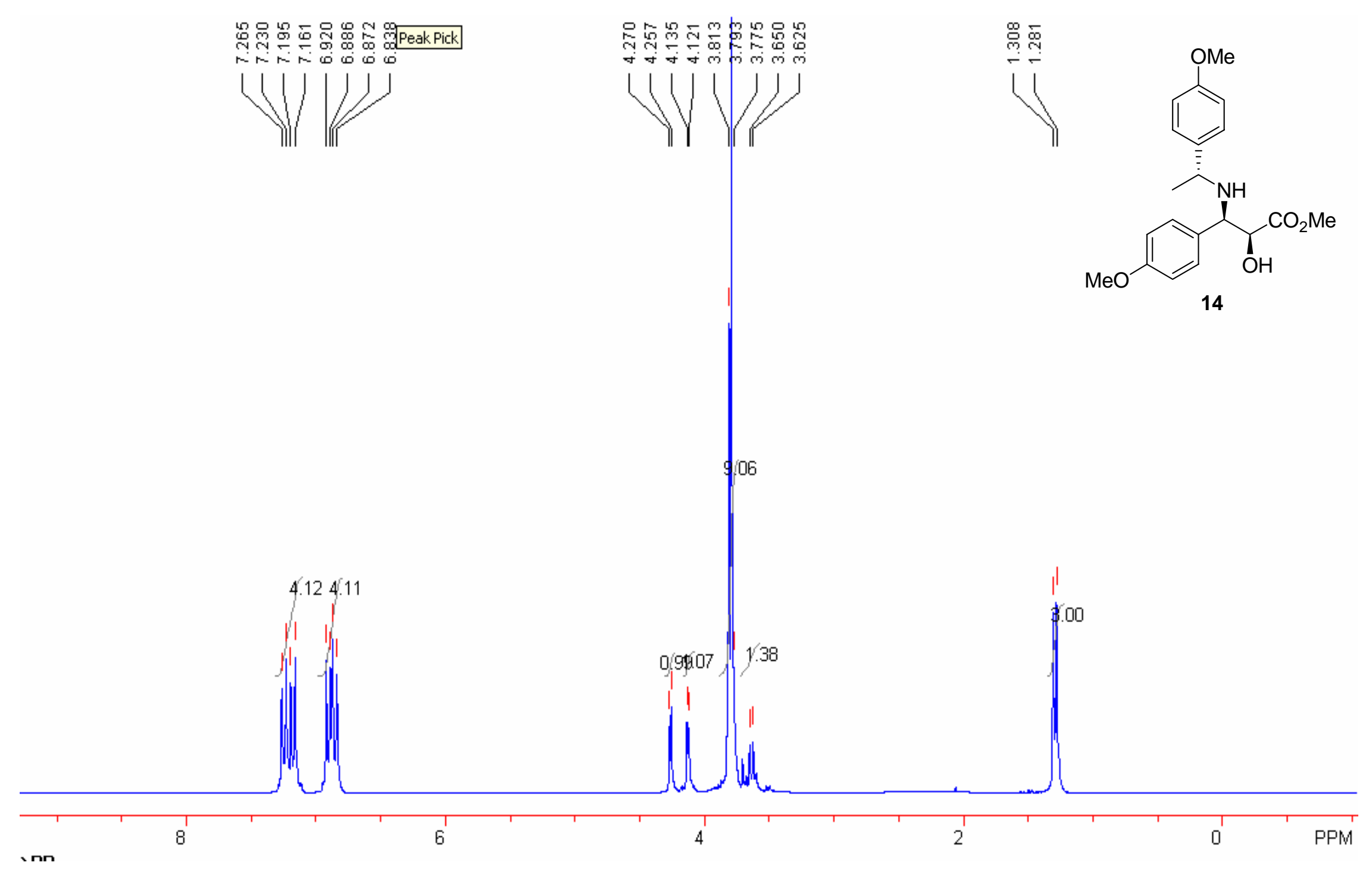




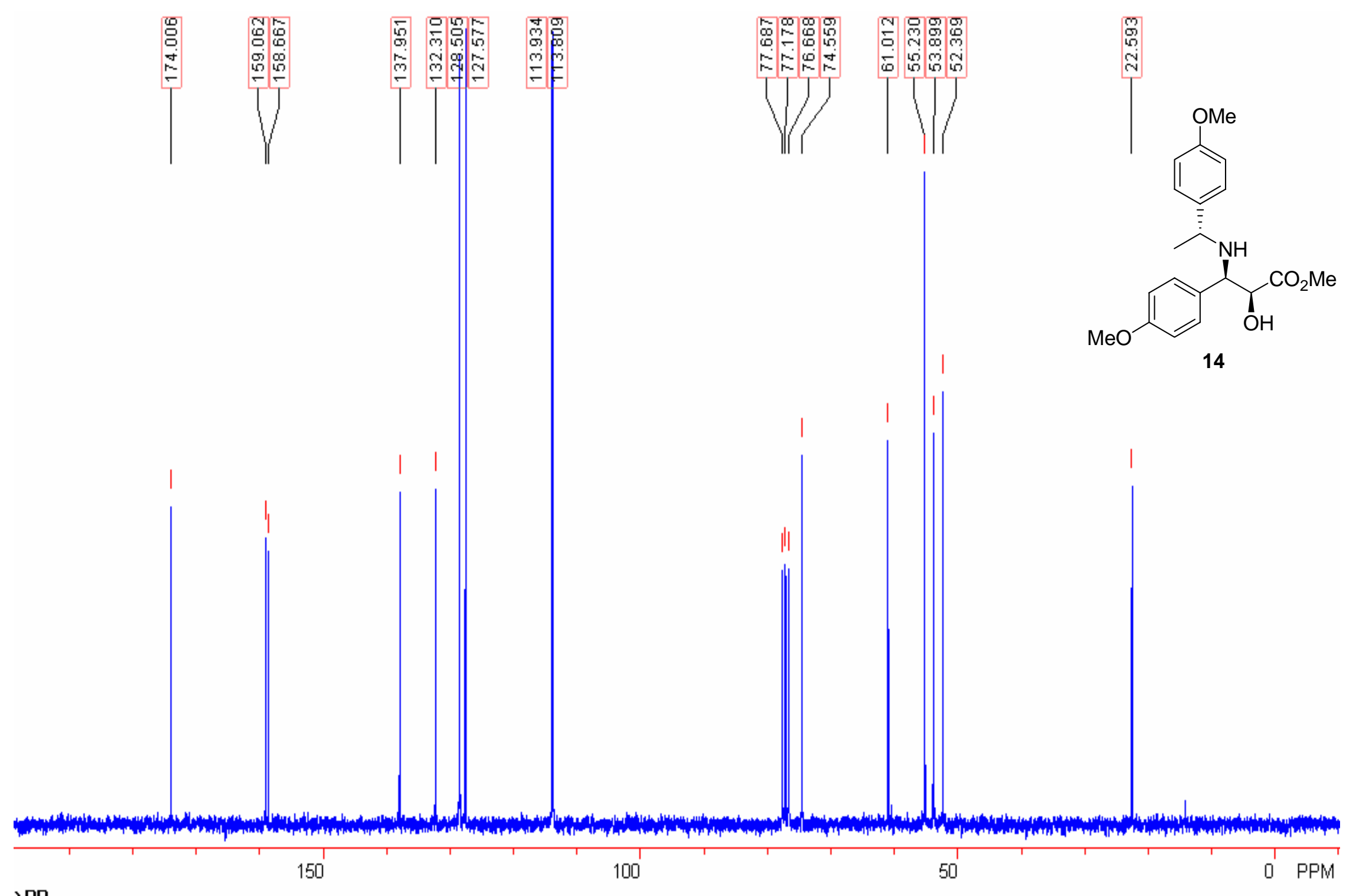




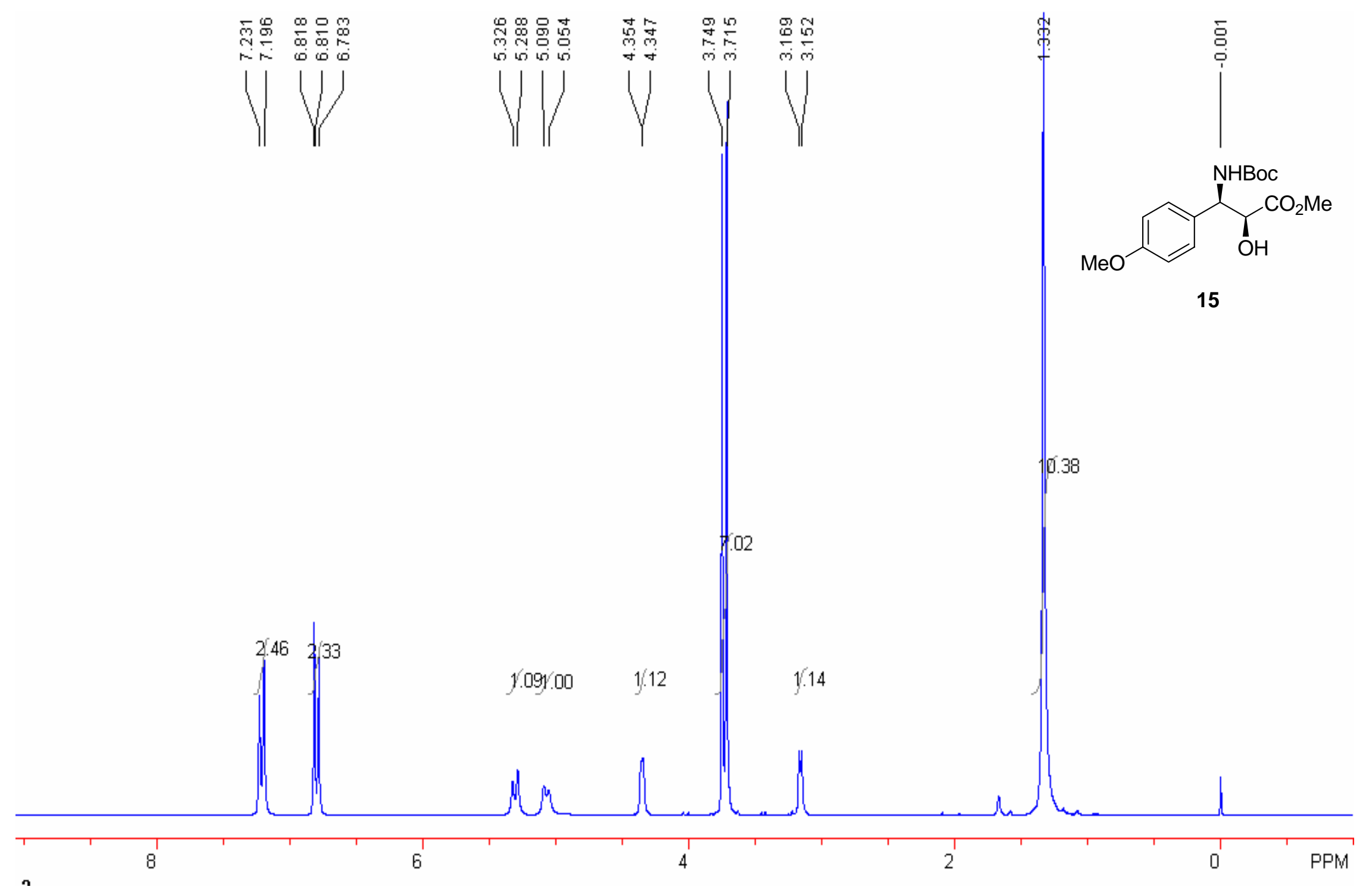



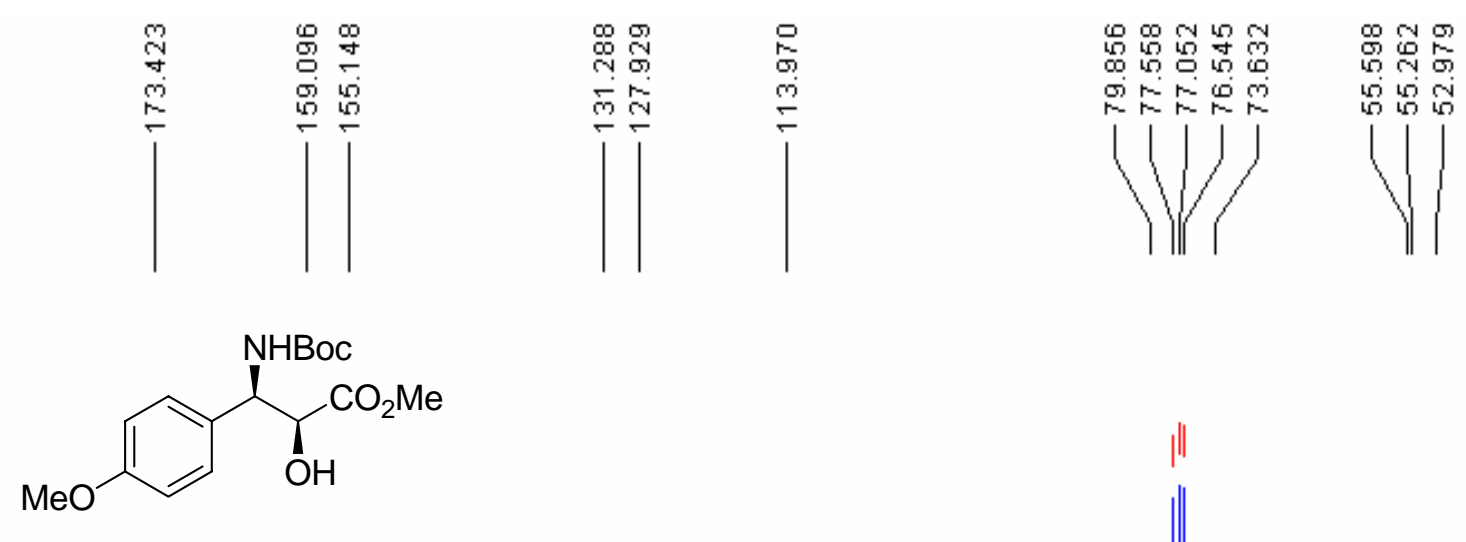

15

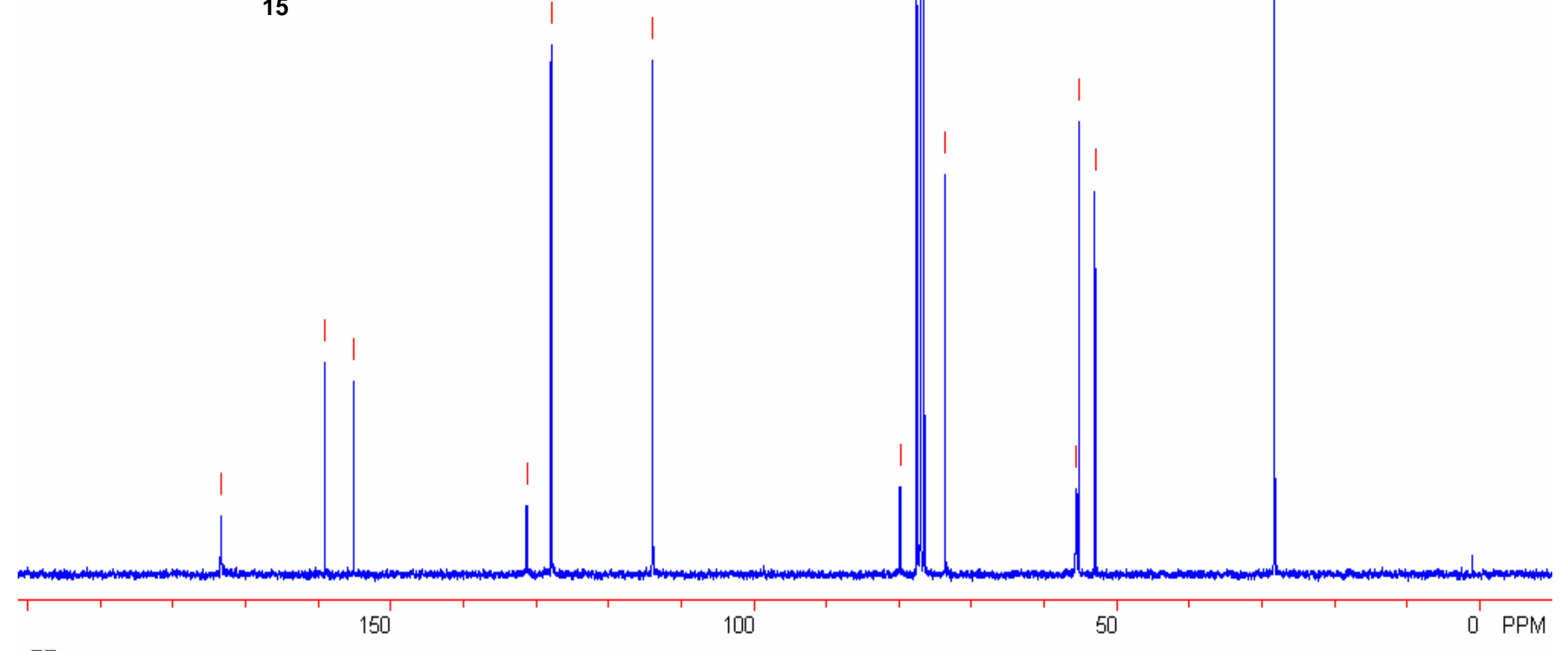



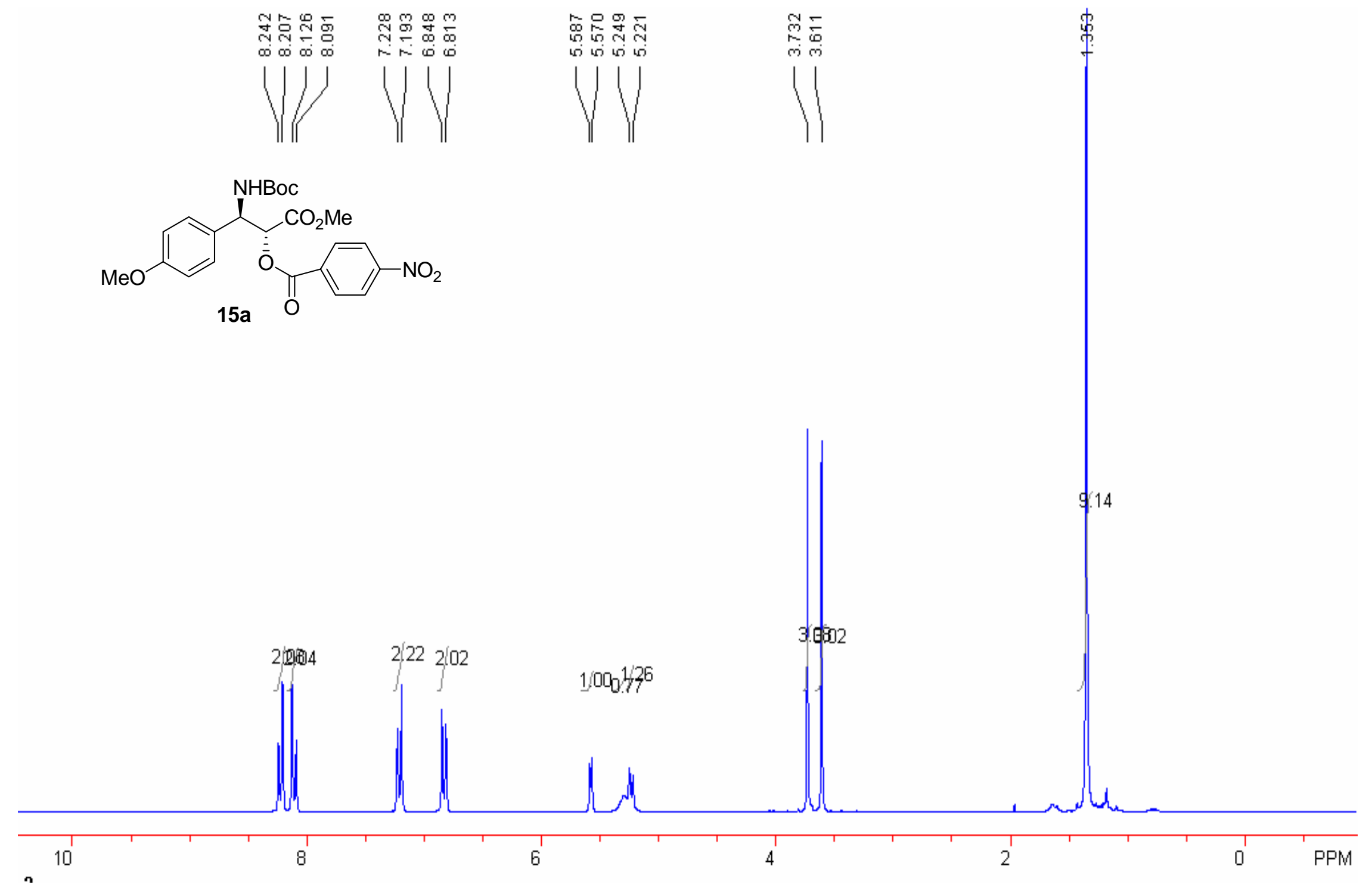

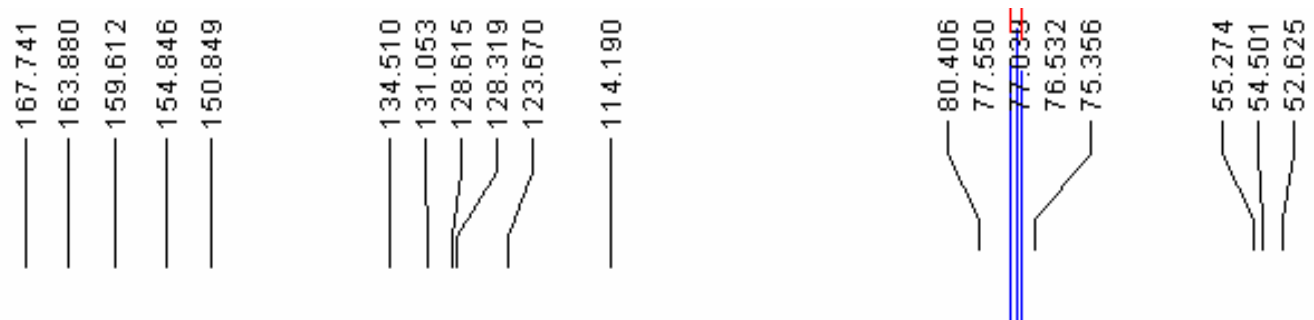

$\overline{9}$

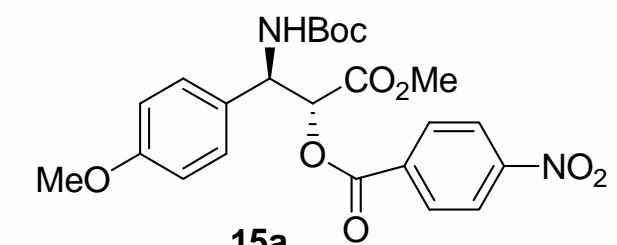

$15 a$
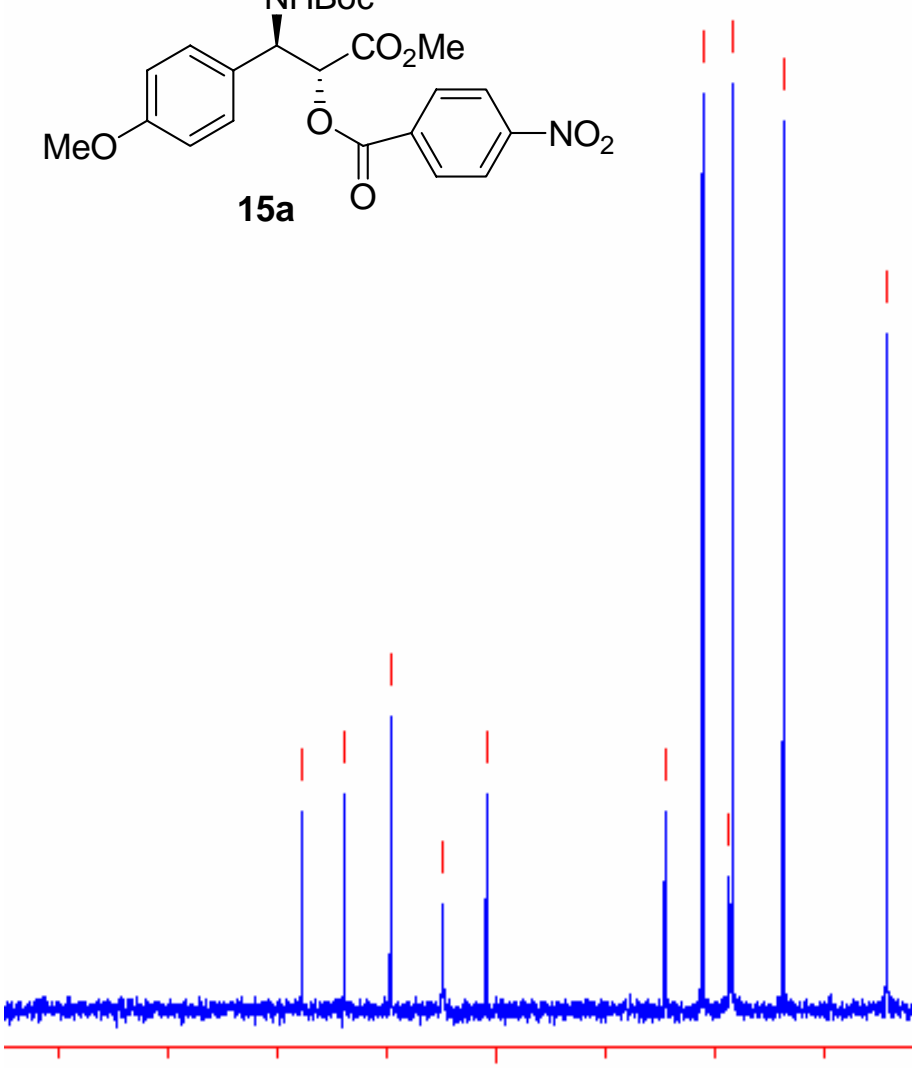

150

100

50

o PPM 


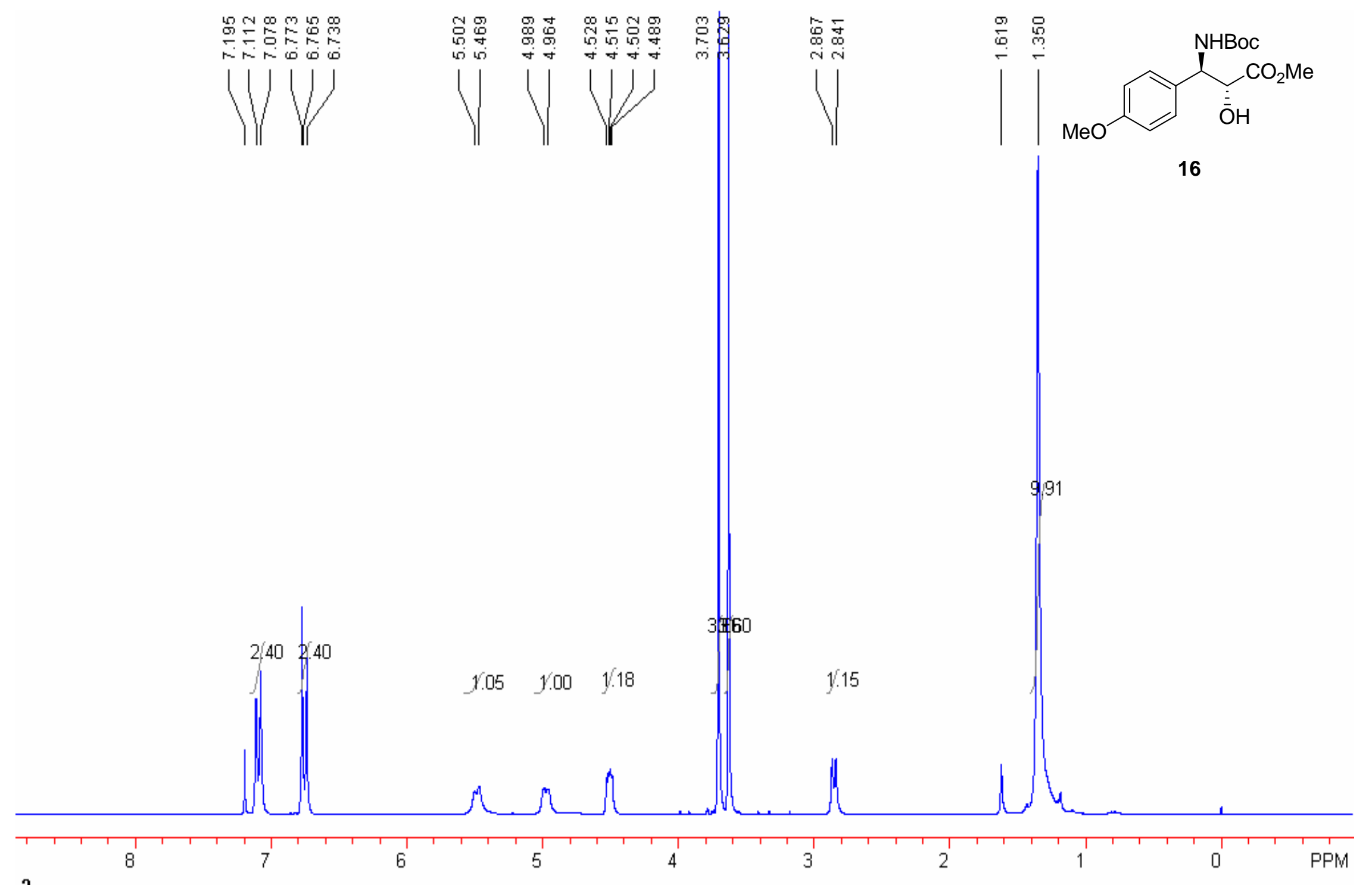


AntiAminolR C13CPD<smiles>COc1ccc([C@H](NC(C)(C)C)C(O)C(C)=O)cc1</smiles>

16

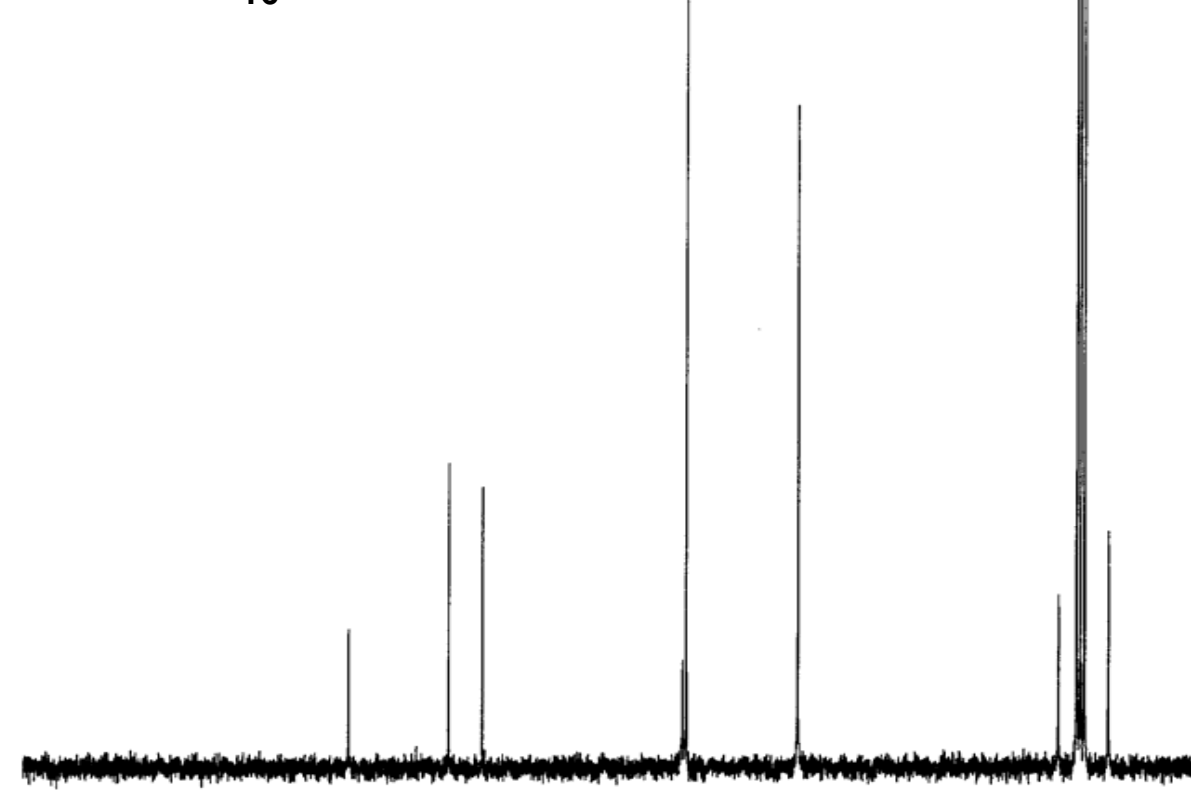

200

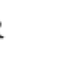

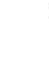



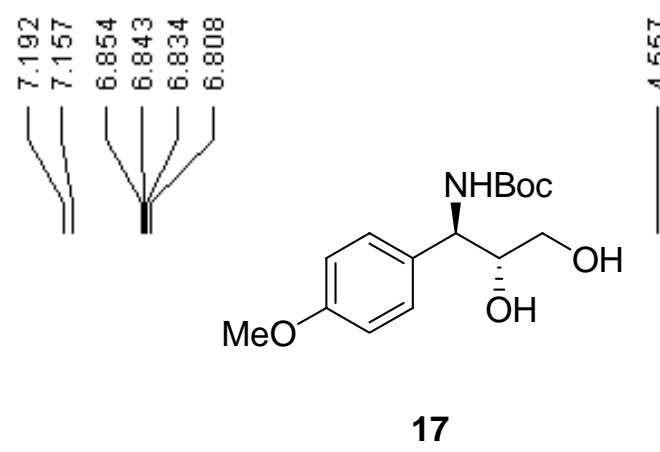

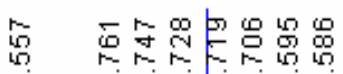

于 लि

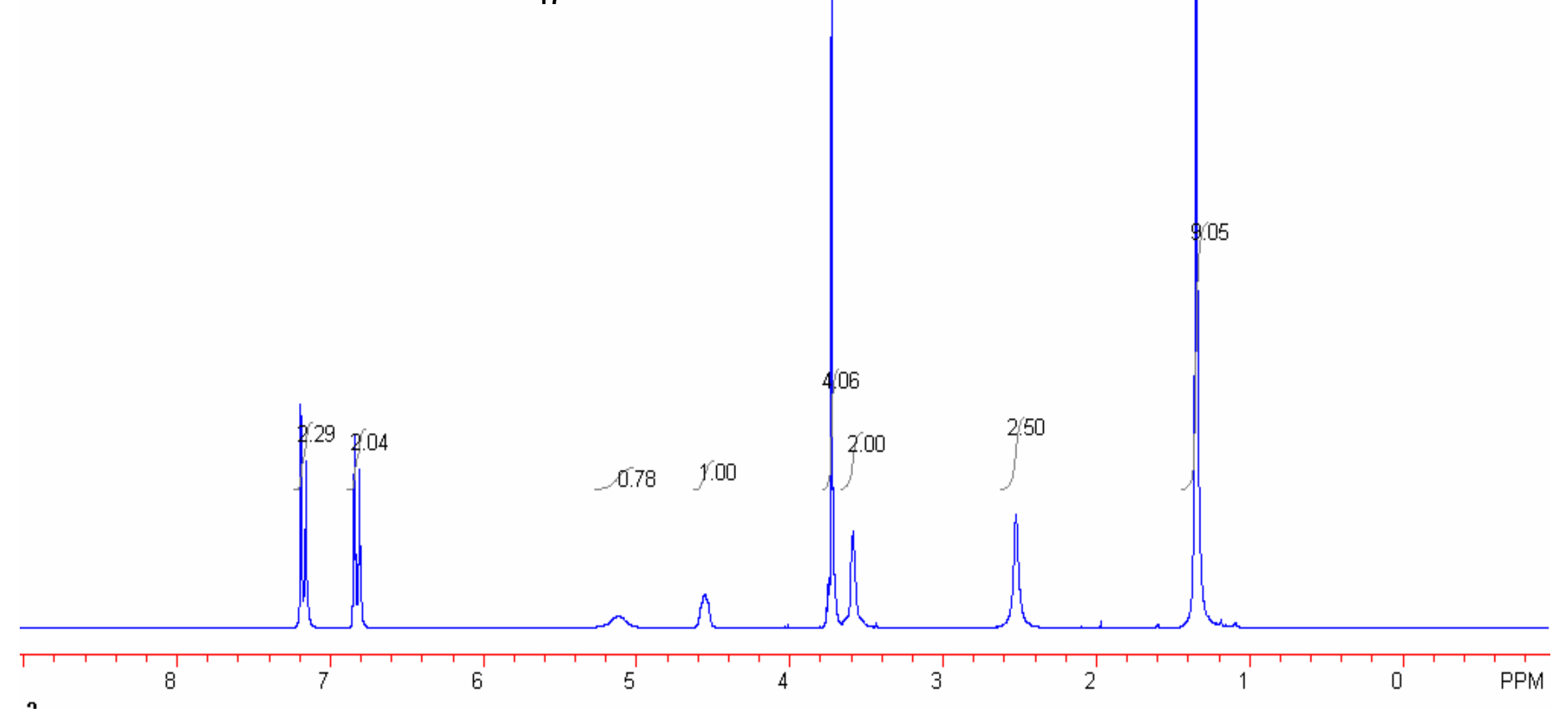




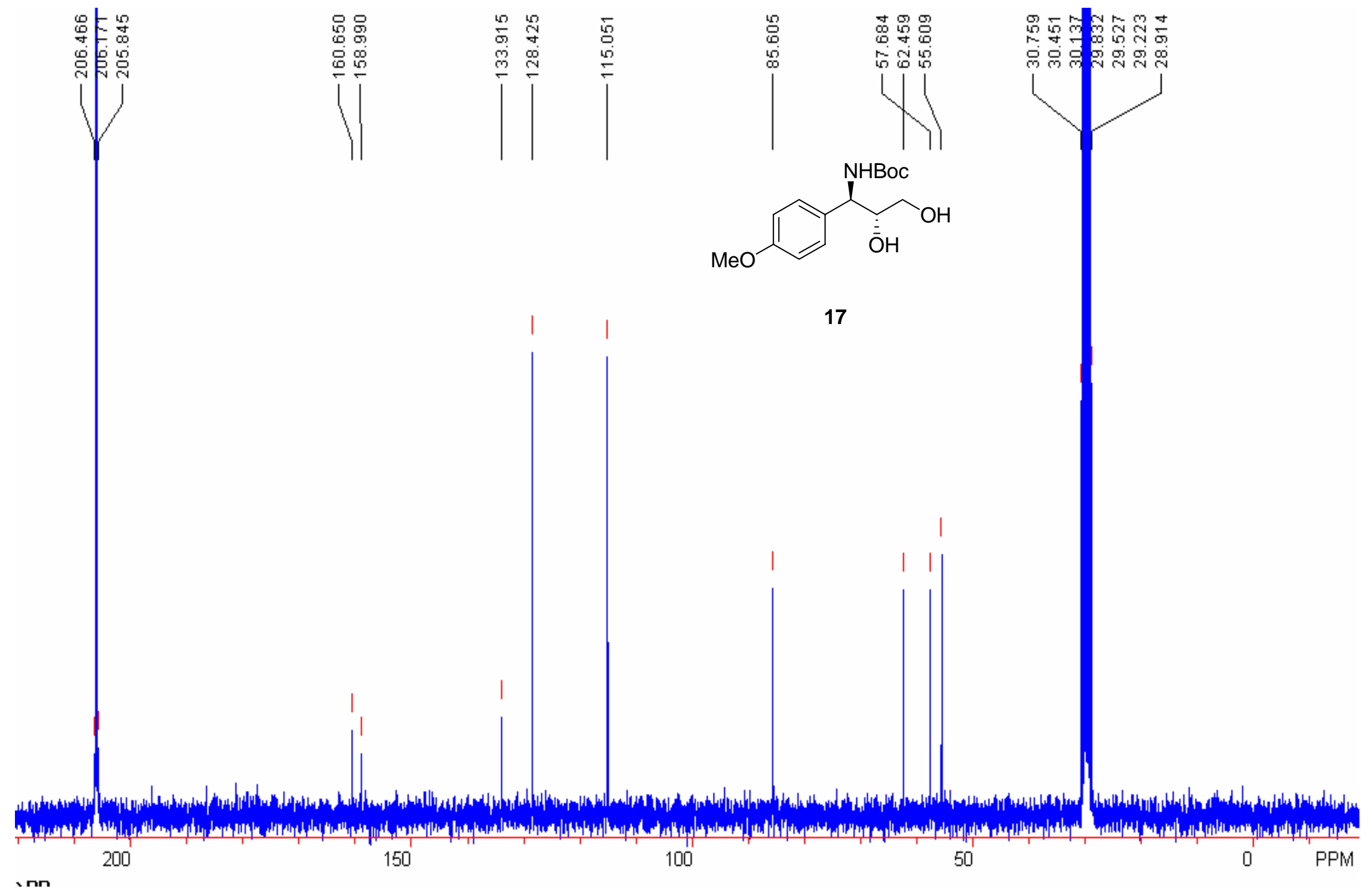




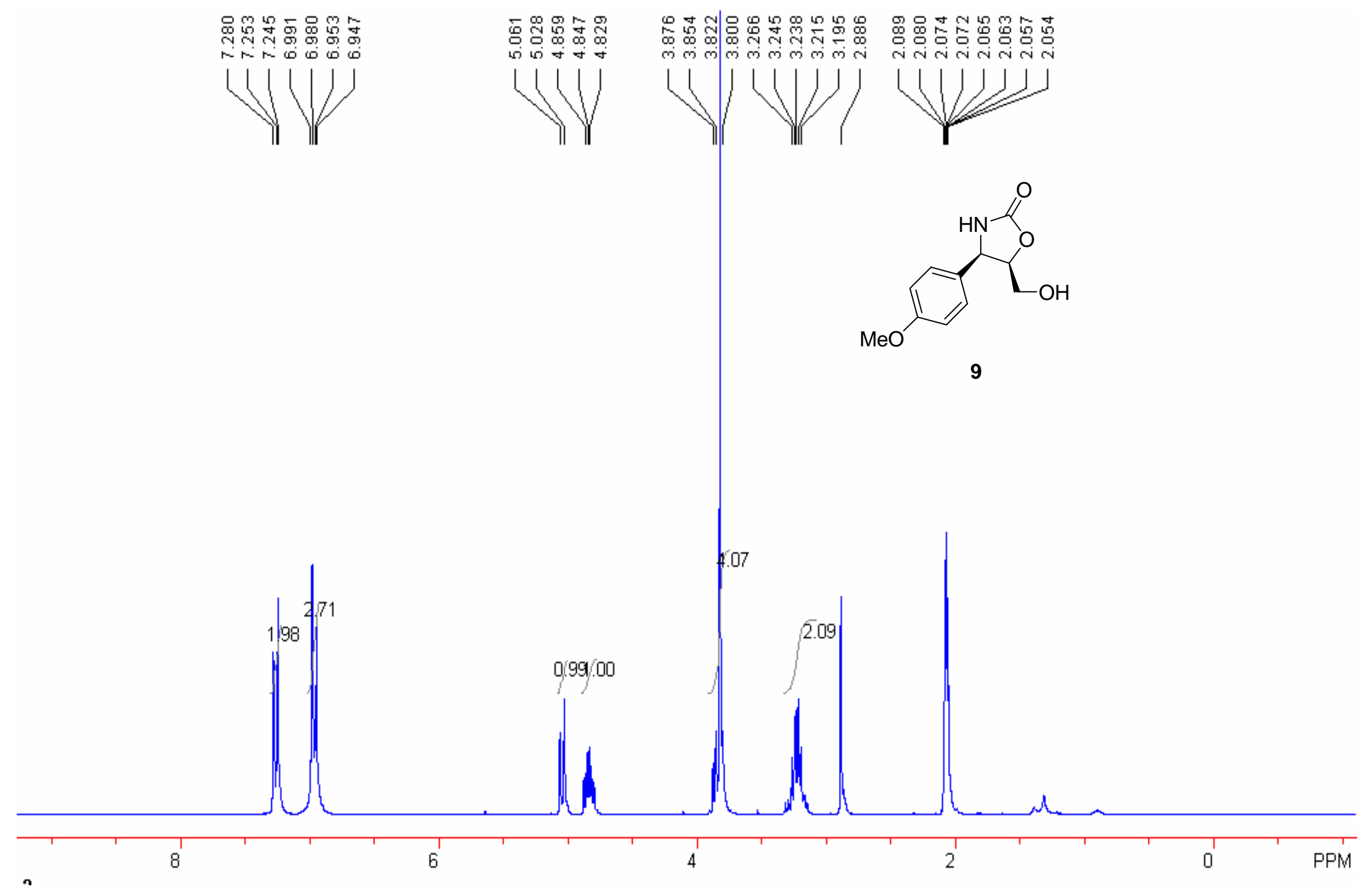




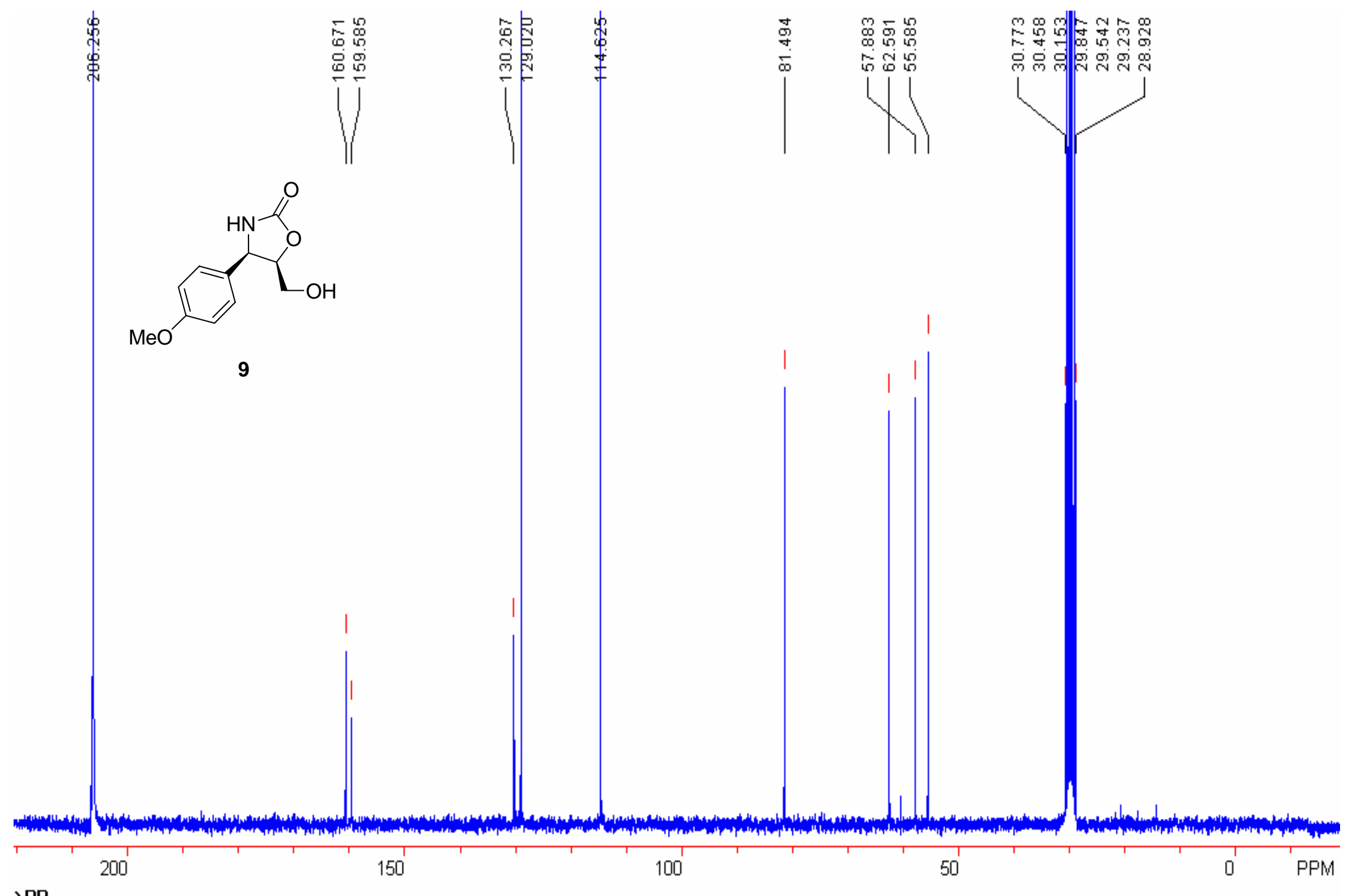




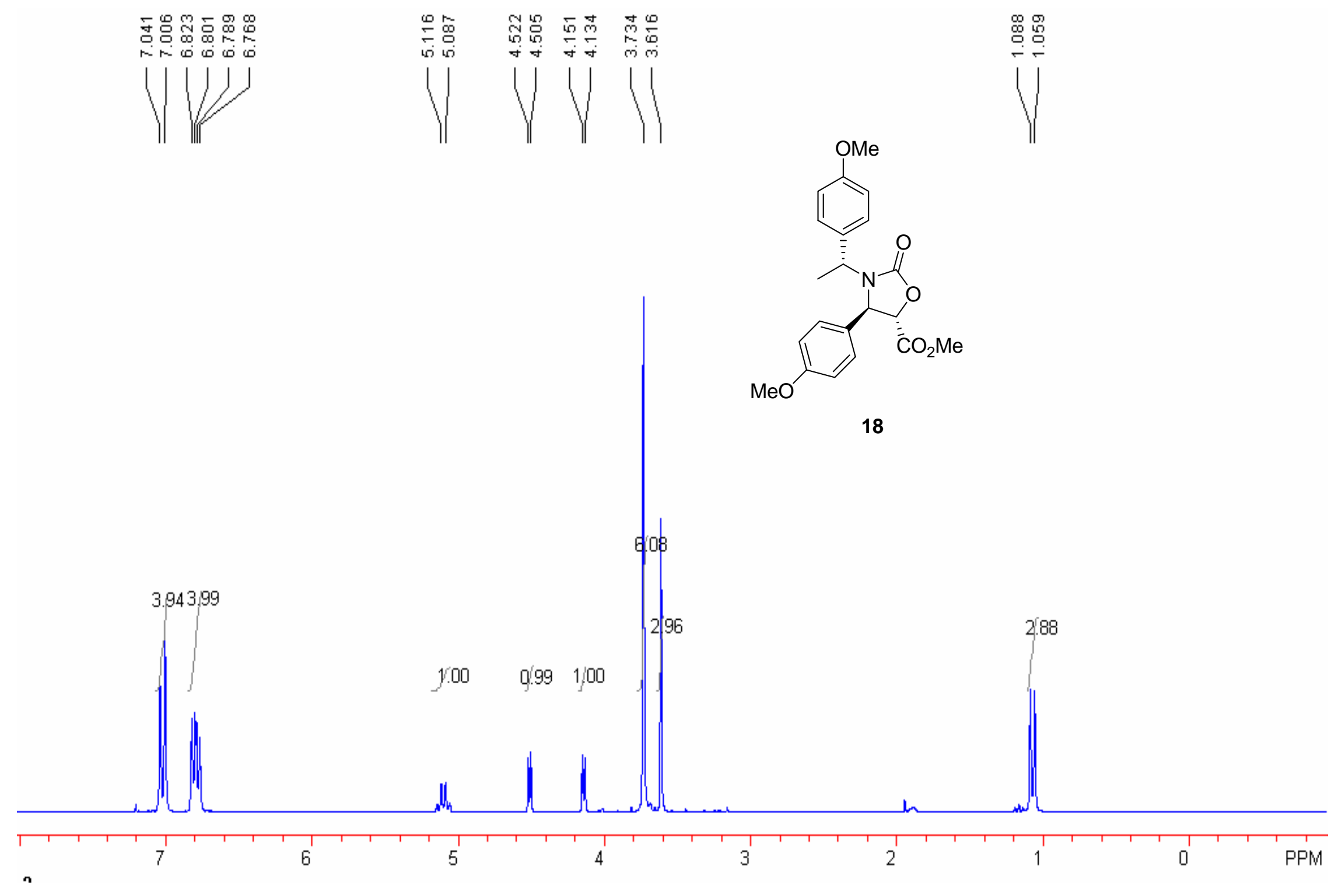




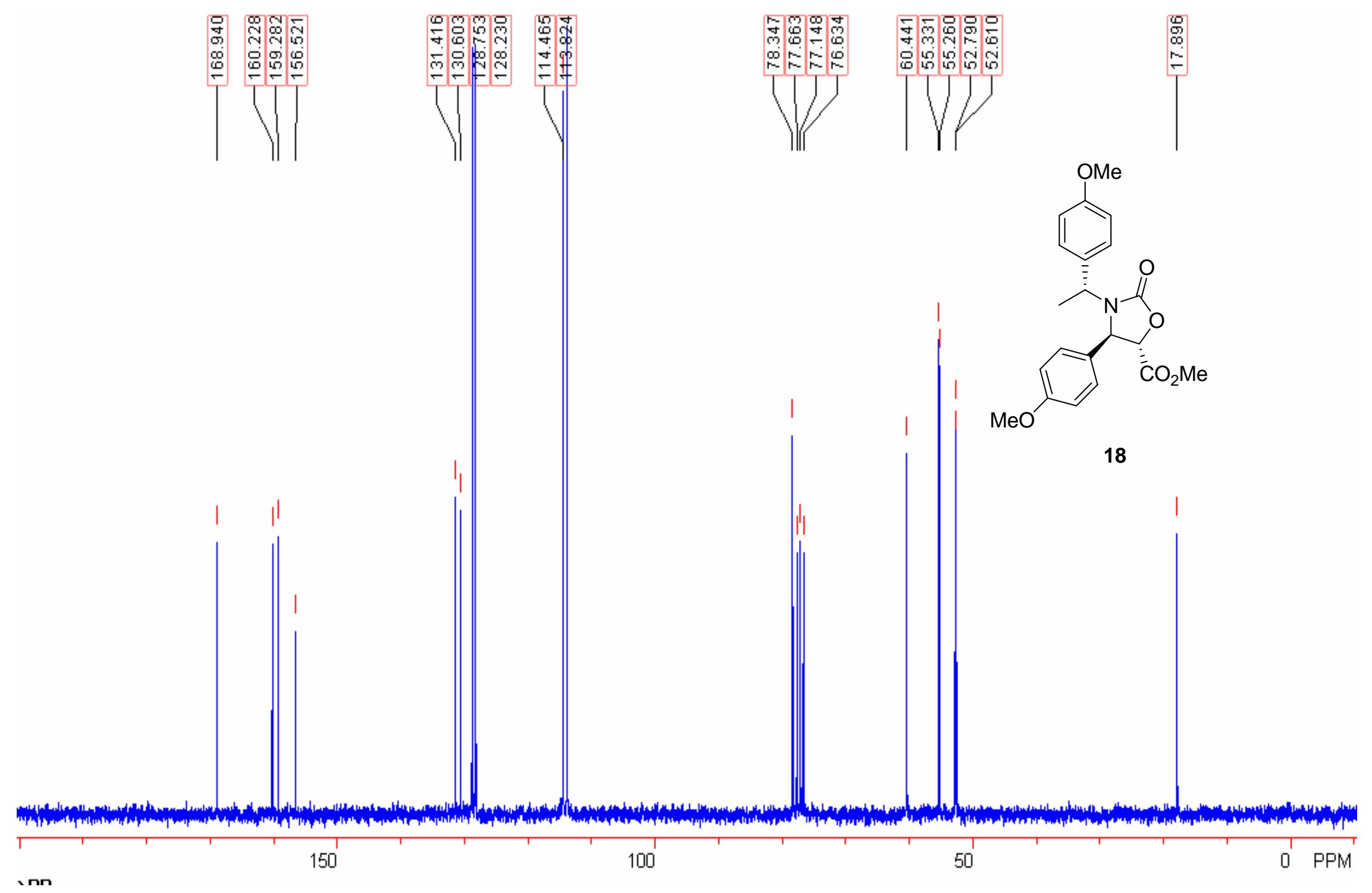




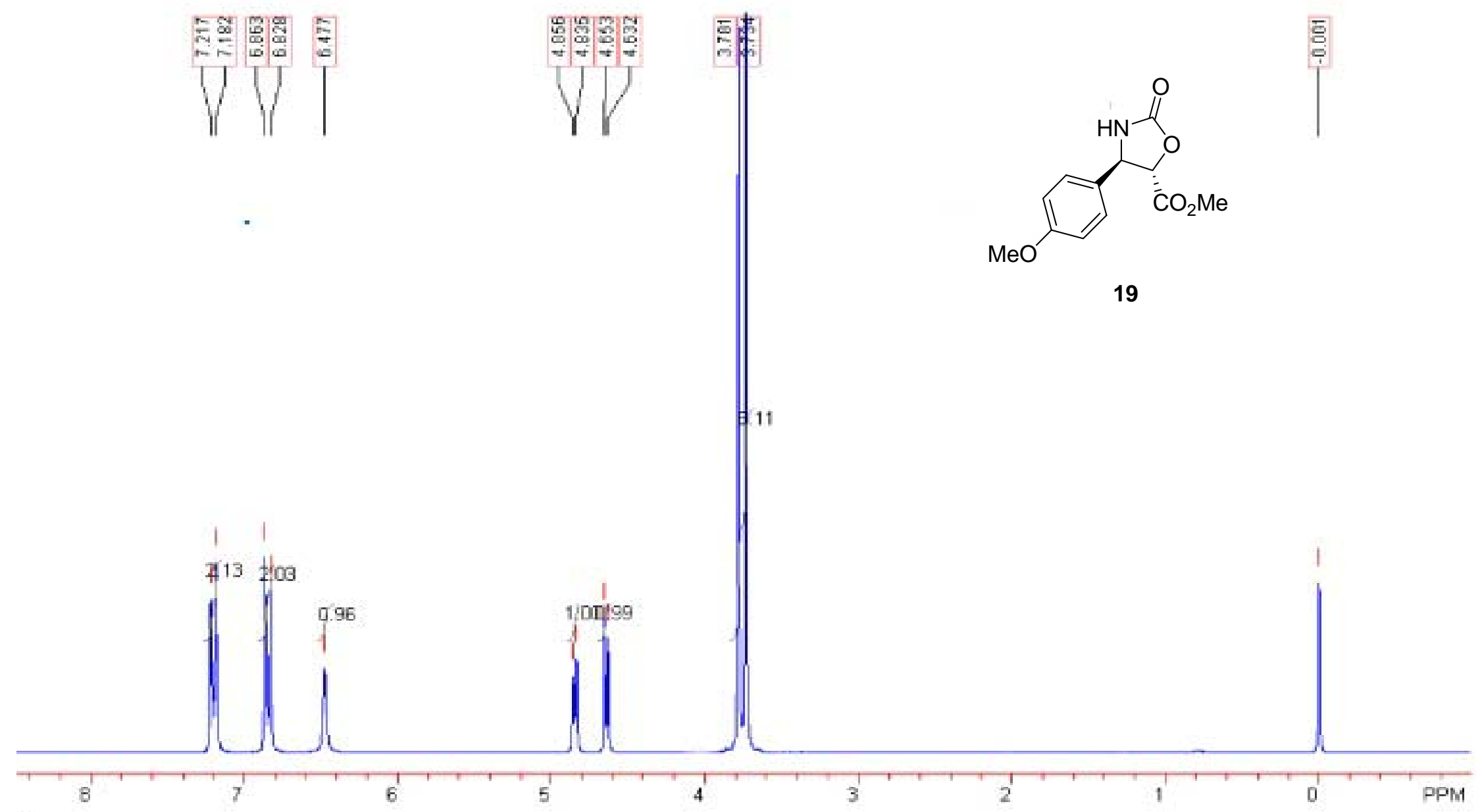




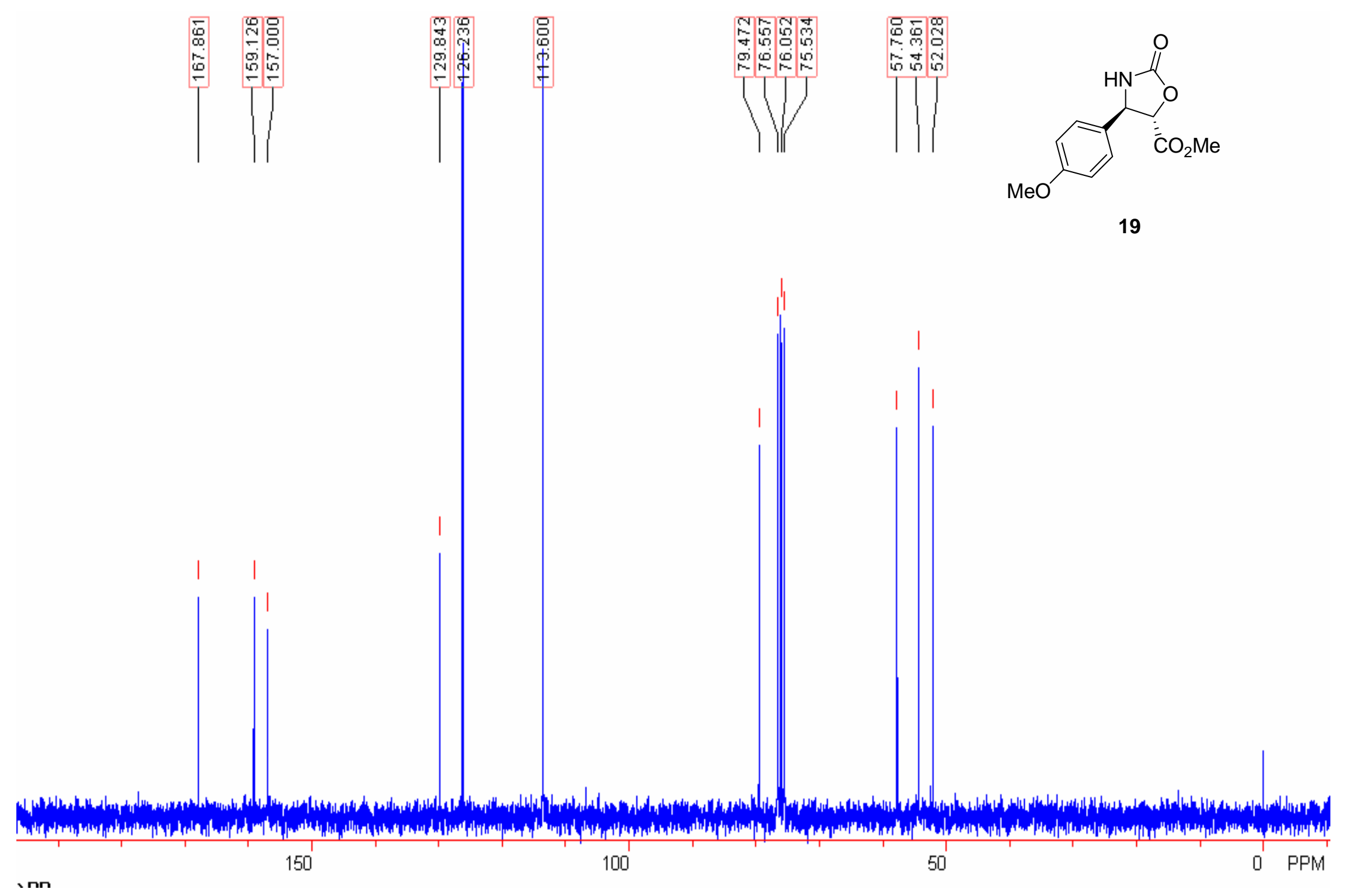


을

$m$ in को

1

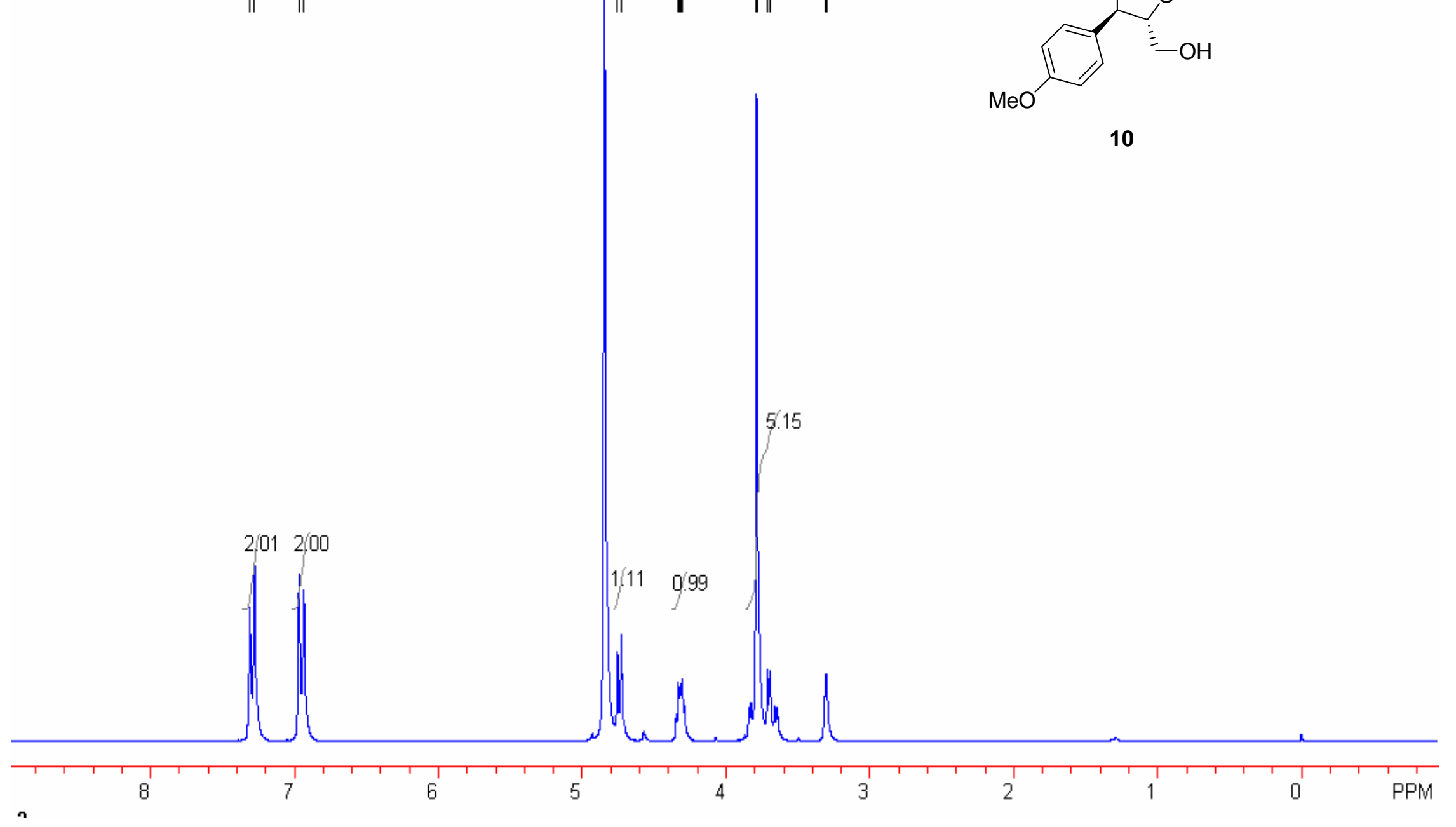

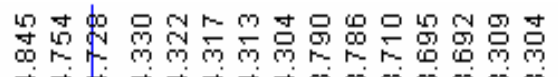
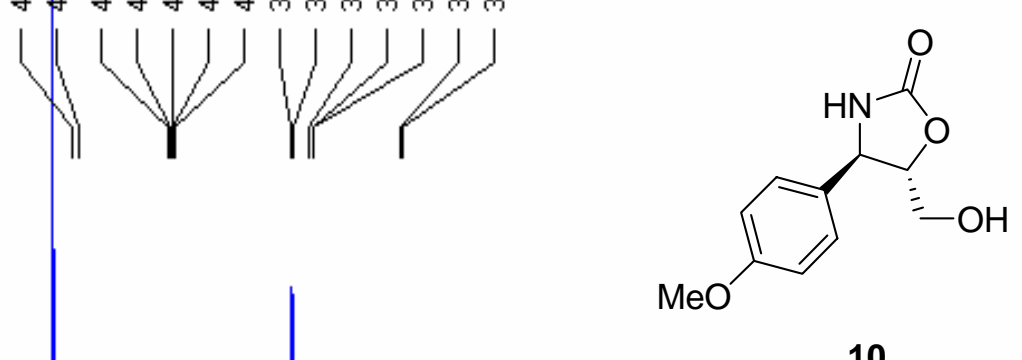

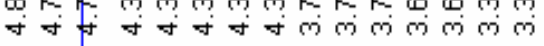




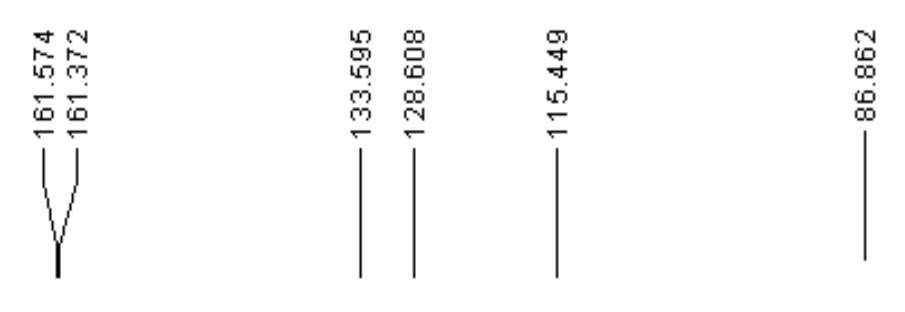

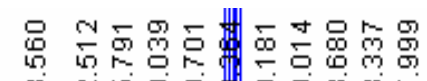

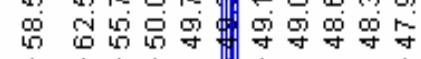

$1+1$
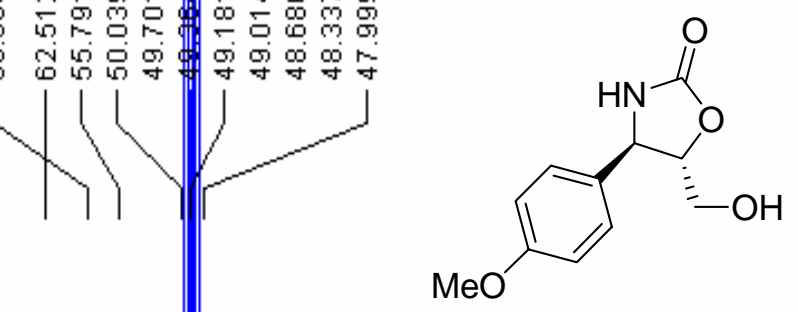

10

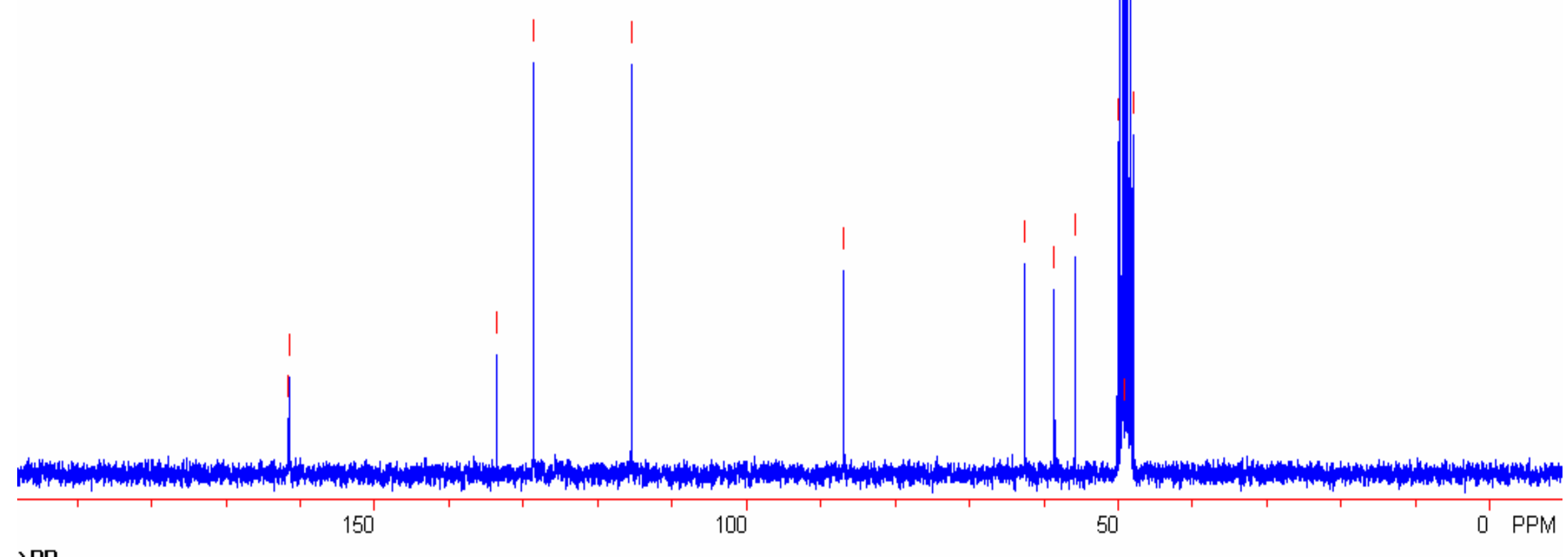

